Article

\title{
Toxicological Activity of Some Plant Essential Oils Against Tribolium castaneum and Culex pipiens Larvae
}

\author{
Ahmed M. El-Sabrout ${ }^{1}$, Mohamed Z. M. Salem ${ }^{2, * \mathbb{D}}$, May Bin-Jumah ${ }^{3} \mathbb{D}$ and Ahmed A. Allam ${ }^{4}$ \\ 1 Department of Applied Entomology and Zoology, Faculty of Agriculture (EL-Shatby), Alexandria University, \\ Alexandria 21545, Egypt; elsabroutahmed@alexu.edu.eg \\ 2 Forestry and Wood Technology Department, Faculty of Agriculture (EL-Shatby), Alexandria University, \\ Alexandria 21545, Egypt \\ 3 Biology Department, College of Science, Princess Nourah bint Abdulrahman University, Riyadh 11671, \\ BO. Box 24428, Saudi Arabia; may_binjumah@outlook.com \\ 4 Department of Zoology, Faculty of Science, Beni-suef University, Beni-suef 65211, Egypt; \\ allam1081981@yahoo.com \\ * Correspondence: zidan_forest@yahoo.com
}

Received: 1 November 2019; Accepted: 4 December 2019; Published: 7 December 2019

\begin{abstract}
In the present work, essential oils (EOs) from Schinus terebinthifolius (ripe and unripe fruits and leaves), Origanum majorana (air-dried aerial parts), and Psidium guajava (leaves) were assayed for their insecticidal activity against red flour beetle (Tribolium castaneum) and Culex mosquito larvae (Culex pipiens). Several components were identified in the EOs using Gas chromatography-mass spectrometry (GC/MS), of which $\Delta$-3-carene (25.9\%), $\gamma$-terpinene (19.4), and $\gamma$-elemene $(7.1 \%)$ were the major ones in S. terebinthifolius ripe fruits, $\alpha$-pinene (48.9\%), germacrene D (12.9\%), and $\alpha$-thujene $(7.7 \%)$ in S. terebinthifolius unripe fruits, $\gamma$-elemene $(11.7 \%)$, spathulenol $(10.1 \%), \beta$-elemene $(9.2 \%)$, and $p$-cymene (9.1\%) in S. terebinthifolius leaves, $\alpha$-pinene (25.5\%), (E)-caryophyllene (15.7\%), (E)-nerolidol (16.7\%), and cedran-8-ol (8.8\%) in P. guajava leaves, and terpinen-4-ol (21.7\%), $\gamma$-terpinene (16.5\%), and sabinene $(10.1 \%)$ in O. majorana air-dried aerial parts. The lethal concentration $\left(\mathrm{LC}_{50}\right)$ was calculated for tested EOs at different time periods (after 6, 12, 24, 48, and $72 \mathrm{~h}$ ). After $6 \mathrm{~h}$ of treatment, the $\mathrm{LC}_{50}$ was 33.3 and $6.8 \mu \mathrm{g} / \mathrm{L}$ air for $S$. terebinthifolius ripe and unripe fruits, respectively, and $>40 \mu \mathrm{g} / \mathrm{L}$ air for EOs of S. terebinthifolius leaves, O. majoranaair-dried aerial parts, and P. guajava leaves. After $24 \mathrm{~h}$ of treatment, the $\mathrm{LC}_{50}$ was $4.2,<2,5,>40$, and $6.1 \mu \mathrm{g} / \mathrm{L}$ air for EOs of $S$. terebinthifolius ripe fruits and leaves, O. majorana leaves, and P. guajava leaves, respectively. On the other hand, the $\mathrm{LC}_{50}$ values decreased when the exposed period was increased to $72 \mathrm{~h}$, and were $<2 \mu \mathrm{g} / \mathrm{L}$ air for EOs of S. terebinthifolius ripe fruits, unripe fruits, and leaves along with P. guajava leaves, respectively, and 37.912 for EO of O. majorana leaves. The $\mathrm{LC}_{50}$ value after $24 \mathrm{~h}$ of exposure of $S$. terebinthifolius unripe fruit $\mathrm{EO}$ was under $2 \mu \mathrm{g} / \mathrm{L}$ air, which means that the EO of $S$. terebinthifolius ripe fruit had a strong effect on adult T. castaneum adults compared to other tested EOs using the fumigation method. The present data confirm that the EOs of O. majorana leaves and S. terebinthifolius unripe fruits and leaves were more effective as larvicide than the EOs of $S$. terebinthifolius ripe fruits and P. guajava leaves on C. pipiens at a higher concentration $(100 \mathrm{mg} / \mathrm{L})$ when applied by the dipping method. EOs from $S$. terebinthifolius unripe or ripe fruits and leaves and P. guajava leaves were more effective as adulticide than EO of O. majorana leaves against T. castaneum when applied by the fumigant method.
\end{abstract}

Keywords: physiological effects; essential oils; Culex mosquitoes; red flour beetle

\section{Introduction}

Botanicals are basically secondary metabolites that serve as a defence mechanism for plants to withstand the continuous selection pressure from herbivore predators and other environmental factors. 
Plants produce essential oils (EOs), terpenoids, alkaloids, steroids, and phenolics in which many have medicinal, insecticidal, and larvicidal activities [1-10]. Plant EOs, in general, have been recognized as an important natural resource for insecticides [6,8,10-15].

In recent years, and due to the lack of novel insecticides and the high cost of synthetic insecticides, the use of many synthetic insecticides formerly used in mosquito control programs has been limited $[5,16,17]$. Therefore, the search for alternatives to synthetic chemical insecticides and natural extracts and EOs for vector and pest management that pose little threat to human and environmental health has increased $[10,18,19]$.

Schinus terebinthifolius Raddi (Sapindales: Anacardiaceae) EOs have been reported to have insecticidal properties against Stegomyia aegypti Linnaeus in Hasselquist (Diptera: Culicidae), Anopheles gambiae sensu lato (Diptera: Culicidae), and Culex quinquefasciatus Say (Diptera: Culicidae) [20]. EOs of mature and immature $S$. terebinthifolius showed strong insecticidal activity against Spodoptera littoralis Boisduval (Lepidoptera: Noctuidae) and Phthorimaea operculella Zeller (Lepidoptera: Gelechiidae) [21]. EO of 1\% S. terebinthifolius fruit showed great repellency against Bemisia tabaci Gennadius (Hemiptera: Aleyrodidae) and potency against Trialeurodes ricini Misra (Homoptera: Aleyrodidae) adults [8]. Leaf extract of S. terebinthifolius caused damage to the midgut of Aedes aegypti Linnaeus in Hasselquist (Diptera: Culicidae) larvae [22]. EOs from fruits and seeds showed mosquitocidal activity against $A n$. gambiae, An. Arabiensis Patton (Diptera: Culicidae), and C. quinquefasciatus [23].

EO obtained from leaves of guava (Psidium guajava L., Myrtales: Myrtaceae) has shown promising larvicidal activity against $A$. aegypti, with $\mathrm{LC}_{50}$ ranging from 39.48 to $64.25 \mu \mathrm{g} / \mathrm{mL}$ [24] and $24.7 \mu \mathrm{g} / \mathrm{mL}$ [25]. P. guajava leaf EO showed notable larvicidal activity against Chaoborus plumicornis F. (Diptera: Chaoboridae) and insecticidal activity against Drosophila melanogaster Meigen (Diptera: Drosophilidae) [26].

Origanum majorana L. (Lamiales: Lamiaceae) EO is composed of majority constituents, which gives it biological activities [27]. The important larvicidal activity observed by the EO of O. majorana could be explained by its chemical composition and the action or effect of the majority compound. Azizi et al. [28] and Pavela [29] reported that Origunum species had insecticidal activity against insects. In general, plant EOs have been recognized as an important natural resource for insecticides [5].

Mosquitoes spread serious human diseases such as malaria, yellow fever, dengue, and filariasis [30]. Overall, 212 million cases of malaria and 429,000 deaths were reported worldwide [31]. In urban and rural of Egyptian areas, C. pipiens L. (Diptera: Culicidae) is the most common mosquito species that causes health risks to humans. The major insects of stored grains and pulses of many countries such as India, Egypt, and others are rice weevil, Sitophilus oryzae L. (Coleoptera: Curculionidae), granary weevil, S. granaries L. (Coleoptera L.: Curculionidae), lesser grain borer, Rhyzopertha dominica Fabricius (Coleoptera: Bostrichidae), Khapra beetle, Trogoderma granarium Everts (Coleoptera: Dermestidae), red flour beetle, Tribolium castaneum Herbst (Coleoptera: Tenebrionidae), saw-toothed grain beetle, Oryzaephilus surinamensis L. (Coleoptera: Silvanidae), and others [12,13,32].

Many researchers have reported that plant parts, EOs, extracts, or powders mixed with grains reduced insect oviposition, egg hatchability, and postembryonic development, inhibited reproduction, and induced mortality of insect eggs and progeny production of stored product insects [33-39]. Most recently, Mentha piperita L. (Lamiales: Lamiaceae) leaf EO at concentrations of 20 and $40 \mu \mathrm{L} / \mathrm{L}$ showed mortality against T. castaneum at $65 \%$ and $90 \%$, respectively [39], with the fumigation method. Additionally, application of Taxodium EOs from different locations in Egypt showed $\mathrm{LC}_{50}$ against $T$. castaneum with values of 66.4 and $72.5 \mu \mathrm{L} / \mathrm{L}$, respectively [13]. EOs of Ocimum basilicum L. (Lamiales: Lamiaceae) and Eucalyptus gomphocephala DC (Myrtales: Myrtaceae) showed larvicidal activity against mosquitos with $\mathrm{LC}_{50}$ values of 22 and $30 \mathrm{mg} / \mathrm{L}$, respectively [10].

The aim of the present study was to evaluate the larvicidal and mosquitocidal activity of five EOs from Schinus terebinthifolius (ripe and unripe fruits and leaves), Origanum majorana (aerial parts), and Psidium guajava (leaves) against C. pipiens by the dipping method. An experiment was also 
conducted to evaluate adulticide activity of the tested EOs against $T$. castaneum using the fumigant method at several concentrations during different exposure times.

\section{Materials and Methods}

\subsection{Plant Materials and Extraction of Essential Oils}

Freshly collected samples (200 g) of ripe and unripe Schinus terebinthifolius fruits, S. terebinthifolius leaves, Origanum majorana air-dried aerial parts, and Psidium guajava leaves were cut into small pieces using scissors and hydro-distilled for $3 \mathrm{~h}$ using a Clevenger-type apparatus [40]. The collected essential oils (EOs) were dried over anhydrous sodium sulphate (Sigma-Aldrich, Darmstadt, Germany). The EO yields were $3.50,2.75,1.15,3.50$, and $0.5 \mathrm{~mL} / 100 \mathrm{~g}$ plant material for $S$. terebinthifolius ripe fruits, S. terebinthifolius unripe fruits, S. terebinthifolius leaves, O. majorana air-dried aerial parts, and P. guajava leaves, respectively.

\subsection{GC-MS Analysis Conditions}

Analysis of the EOs was performed using an Agilent 6890 gas chromatograph-mass spectrometer (GC-MS) equipped with an Agilent mass spectrometry detector with a direct capillary interface and HP-5MS fused silica capillary column $(30 \mathrm{~m} \times 0.32 \mathrm{~mm} \times 0.25 \mu \mathrm{m}$ film thickness) (Thermo Scientific, Austin, TX, USA). The program temperature and samples were carried out following previous published works [41,42]. Identification of the constituents was performed based on an mass spectra (MS) library search (National Institute of Standards and Technology (NIST) and Wiley), and by comparing with data in the MS literature $[43,44]$. The EO compounds were confirmed using the Xcalibur 3.0 data system (3.0, Thermo Fisher Scientific Inc., Austin, TX, USA, 2014) with measuring their Standard Index and Reverse Standard Index [45-48].

\subsection{Red Flour Beetle Rearing}

Red flour beetle (T. castaneum) adults and larvae were reared on wheat flour under laboratory conditions of $27 \pm 3{ }^{\circ} \mathrm{C}$ and $70 \pm 5 \%$ relative humidity (RH).

\subsection{Mosquito Rearing}

A susceptible strain of mosquito larvae, C. pipiens, was obtained from the Research Institute of Medical Entomology, Dokki, Egypt. The continuously breeding mosquito colony was maintained in an insectary at $27 \pm 2{ }^{\circ} \mathrm{C}, 75 \pm 5 \% \mathrm{RH}$ at the Department of Applied Entomology and Zoology, Alexandria University, Egypt. The rearing of larvae and feeding of adults were done according to the method of Zahran and Abdelgaleil [30] with some modification.

\subsection{Fumigant Assay on Red Flour Beetle}

The fumigation experiment was carried out at $26 \pm 1{ }^{\circ} \mathrm{C}$ and $65 \pm 5 \% \mathrm{RH}$. Newly emerged adults (1-15 days old) were used in fumigant studies. The fumigant method for the 5 EOs was tested against T. castaneum adults. Glass jars ( $1 \mathrm{~L})$ were used as fumigation chambers (replicates) and filter paper pieces $(3 \times 3 \mathrm{~cm})$ were joined to the undersurface of the screw caps of the jars. The 5 EOs were applied to the filter paper pieces by $2,5,10,20$, and $40 \mu \mathrm{L} / \mathrm{L}$ air. Every jar as a replicate containing 20 insects as treatment and control were repeated 3 times. Filter paper pieces were treated with acetone (Loba Chemie Pvt. Ltd., laboratory reagents \& fine chemicals, Mumbai, India) alone as a control. Control insects were kept under the same conditions with acetone. The insect mortality percentage was observed after $6,24,48$, and $72 \mathrm{~h}$ of treatment and the lethal concentration causing $50 \%$ mortality $\left(\mathrm{LC}_{50}\right)$ expressed as $\mathrm{mg} / \mathrm{L}$ air was calculated from log-concentration mortality regression lines. Insects were considered dead when no leg or antenna movements were recorded. The fumigant method assay was performed as described by Finney [49], El-Bakry et al. [50], and Huang et al. [51]. 


\subsection{Bioassay Toxicity of Mosquitos}

The tested EOs of $S$. terebinthifolius ripe and unripe fruits and leaves, $O$. majorana air-dried aerial parts, and P. guajava leaves were examined for bioassays [30] on newly second instar larvae of $C$. pipiens. This experiment was conducted by the dipping method using four concentrations of each oil $(10,25$, 50 , and $100 \mathrm{mg} / \mathrm{L}$ ). Three replicates for each concentration were prepared. Each replicate including 50 C. pipiens larvae was separately put into a $200-\mathrm{mL}$ plastic cup containing $100 \mathrm{~mL}$ of distilled water. The tested EO solutions were added to the cups and suspended with $0.05 \mathrm{~mL}$ of Tween-20.

The C. pipiens larvae were exposed to 10, 25, 50, and $100 \mathrm{mg} / \mathrm{L}$ of tested EOs in $100 \mathrm{~mL}$ of distilled water. In the control cups, only solvent (absolute acetone) was dissolved in the water. Treated and control larvae were held in the same conditions used for colony rearing. Larval mortality was recorded 24 and $48 \mathrm{~h}$ after treatment and continued to the end of the larval stage. Larvae were considered dead when they did not rise to the surface of the solution or when they did not respond to a stimulus. Additionally, pupal and adult mortality was calculated. The longevity parameter was calculated for each development stage of $C$. pipiens.

\subsection{Statistical Analysis}

The mortality data were subjected to probit analysis to estimate the lethal concentration $\left(\mathrm{LC}_{50}\right)$ values of tested EOs. Data for the mortality percentage of $T$. castaneum as affected by 3 factors of different concentrations of 5 EOs with different time periods were statistically analyzed using factorial design. To study the significance effects of oil concentration and oil source as well as their interaction as insecticidal activity against $C$. pipiens, two-way analysis of variance (ANOVA) with a two-factor test was used. All analyses were done using the SAS system (Release 8.02, SAS Institute: Cary, NC, USA, 2001) [52]. Comparisons among means were recorded using $\mathrm{LSD}_{0.05}$.

\section{Results}

\subsection{Chemical Composition of Essential Oils}

Table 1 shows the chemical composition of the essential oils (EOs) from ripe/unripe fruits and leaves of $S$. terebinthifolius. The major components of the oils were $\Delta$-3-carene (25.95\%), $\gamma$-terpinene $(19.45 \%), \gamma$-elemene $(7.07 \%), \alpha$-ylangene $(5.34 \%), p$-cymene $(4.55 \%)$, and $D$-limonene $(2.94 \%)$ in ripe fruits, $\alpha$-pinene $(48.96 \%)$, germacrene $\mathrm{D}(12.95 \%), \alpha$-thujene $(7.75 \%)$, sabinene $(4.96 \%)$, and $D$-limonene $(4.32 \%)$ in unripe fruits, and $\gamma$-elemene (11.74\%), spathulenol (10.13\%), $\beta$-elemene $(9.24 \%), p$-cymene $(9.19 \%), \beta$-phellandrene (5.93\%), naphthalene (5.47\%), and $D$-limonene $(2.22 \%)$ in leaves. The main compounds in EO of P. guajava leaves were $\alpha$-pinene (25.51\%), (E)-caryophyllene (15.74\%), (E)-nerolidol $(16.75 \%)$, and cedran-8-ol (8.81\%) (Table 2). The main compounds in EO of O. majorana air-dried aerial parts were terpinen-4-ol (21.74\%), $\gamma$-terpinene $(16.5 \%)$, sabinene $(10.14 \%), \gamma$-terpinyl acetate $(6.76 \%)$, $\alpha$-terpinene (6.19\%), terpinolene (5.72\%), and $\alpha$-terpineol (5.14\%) (Table 3). 
Table 1. Chemical composition of essential oils from S. terebinthifolius ripe/unripe fruits and leaves.

\begin{tabular}{|c|c|c|c|}
\hline Compound Name & $\begin{array}{c}\text { S. terebinthifolius Ripe } \\
\text { Fruit Oil }\end{array}$ & $\begin{array}{l}\text { S. terebinthifolius } \\
\text { Unripe Fruit Oil }\end{array}$ & $\begin{array}{l}\text { S. terebinthifolius } \\
\text { Leaf Oil }\end{array}$ \\
\hline$\alpha$-Pinene & - & $48.9(696-696)$ & 4.1 (933-933) \\
\hline$\Delta$-3-Carene & $25.9(675-689)$ & - & - \\
\hline$\beta$-Pinene & - & - & 0.7 (880-888) \\
\hline Terpinen-4-ol & - & - & $0.2(869-876)$ \\
\hline$\gamma$-Terpinene & $19.4(657-709)$ & $1.8(818-821)$ & - \\
\hline$\alpha$-Thujene & - & $7.7(670-736)$ & 0.5 (907-929) \\
\hline D-Limonene & 2.9 (918-919) & $4.3(838-840)$ & $2.2(921-928)$ \\
\hline Sabinene & - & $4.9(861-873)$ & - \\
\hline$\beta$-Phellandrene & $1.2(868-871)$ & - & $5.9(833-835)$ \\
\hline$p$-Cymene & $4.5(889-892)$ & - & $9.1(890-890)$ \\
\hline Terpinolene & $1.3(891-894)$ & $1.8(912-913)$ & $0.4(892-915)$ \\
\hline Cymene & - & $2.9(889-890)$ & - \\
\hline Linalool & - & - & 0.5 (896-905) \\
\hline$\alpha, 2$-Dimethyl styrene & - & - & $0.4(897-926)$ \\
\hline Carvenone & $0.3(777-788)$ & $0.1(780-790)$ & $0.7(821-842)$ \\
\hline$\alpha$-Terpineol & - & - & 0.5 (903-919) \\
\hline trans-Piperitol & - & - & - \\
\hline cis-Sabinol & $0.6(911-920)$ & 0.3 (904-915) & 0.7 (869-890) \\
\hline p-Cymen-8-ol & - & - & $0.2(863-902)$ \\
\hline$\gamma$-Terpinyl acetate & - & - & - \\
\hline$\Delta$-Elemene & $2.1(866-873)$ & $1.8(871-899)$ & $0.5(884-888)$ \\
\hline Naphthalene & - & - & $5.4(745-847)$ \\
\hline$\gamma$-Muurolene & $0.45(734-757)$ & $0.21(728-736)$ & $0.3(825-837)$ \\
\hline$\beta$-Elemene & - & - & $9.2(896-899)$ \\
\hline Citronellyl acetate & $1.1(807-847)$ & $0.4(748-805)$ & - \\
\hline Aromandendrene & - & - & $3.9(780-789)$ \\
\hline Neryl acetate & - & - & - \\
\hline$\alpha$-Ylangene & $5.3(853-856)$ & $2.3(854-859)$ & $0.1(781-787)$ \\
\hline$\gamma$-Elemene & $7.1(813-848)$ & $3.7(830-853)$ & 11.7 (892-895) \\
\hline IsoGermacrene-D & $0.9(883-896)$ & 0.7 (885-893) & $0.6(911-868)$ \\
\hline$\gamma$-Cadinene & $1.4(817-837)$ & $0.9(805-850)$ & $0.3(848-887)$ \\
\hline$\gamma$-Selinene & - & - & $0.3(877-879)$ \\
\hline Germacrene D & $14.7(893-894)$ & $12.9(886-889)$ & $3.4(908-911)$ \\
\hline$\beta$-Selinene & - & - & 1.7 (912-933) \\
\hline$\beta$-Copaene & $1.1(855-867)$ & $0.6(850-864)$ & $0.2(868-883)$ \\
\hline Valencene & $0.3(831-844)$ & - & - \\
\hline (+)-Lepidozene & - & $0.2(830-886)$ & $2.1(831-898)$ \\
\hline
\end{tabular}


Table 1. Cont.

\begin{tabular}{|c|c|c|c|}
\hline Compound Name & $\begin{array}{l}\text { Fruit Oil } \\
\text { Fruit Oius Ripe }\end{array}$ & $\begin{array}{l}\text { S. terebinthifolius } \\
\text { Unripe Fruit Oil }\end{array}$ & $\begin{array}{l}\text { S. terebinthifolius } \\
\text { Leaf Oil }\end{array}$ \\
\hline$\Delta$-Cadinene & 0.7 (903-908) & $0.2(869-892)$ & $1.1(906-914)$ \\
\hline Guaiene & - & - & $1.6(827-828)$ \\
\hline Selina-3,7(11)-diene & - & - & $0.4(840-868)$ \\
\hline Calamenene & - & - & $0.3(793-897)$ \\
\hline $\begin{array}{c}\text { trans-Sesquisabinene } \\
\text { hydrate }\end{array}$ & - & $0.1(733-831)$ & - \\
\hline Globulol & - & - & $0.5(34-863)$ \\
\hline Elemoyl acetate & $0.2(830-870)$ & $0.1(797-817)$ & - \\
\hline$\alpha$-Costol & - & - & $0.4(702-756)$ \\
\hline 4(15),5,10(14)-Germacratrien-1-ol & $0.3(763-772)$ & $0.2(816-822)$ & $1.4(804-816)$ \\
\hline$\alpha$-Calacorene & - & - & $0.1(788-923)$ \\
\hline Spathulenol & $1.2(872-895)$ & $0.3(867-869)$ & $10.1(832-852)$ \\
\hline 4(14)-Salvialen-1-one & $0.2(800-851)$ & - & - \\
\hline Rosifoliol & - & - & $0.7(782-873)$ \\
\hline$\beta$-Neoclovene & - & $0.1(788-796)$ & - \\
\hline Eudesma-4,11-dien-2-ol & $0.5(794-800)$ & $0.1(761-767)$ & $0.1(774-800)$ \\
\hline Cubebol & - & - & $0.1(765-822)$ \\
\hline Isospathulenol & $2.3(872-876)$ & $1.1(844-849)$ & $3.5(865-876)$ \\
\hline $\begin{array}{l}\text { Isoaromadendrene } \\
\text { epoxide }\end{array}$ & - & $0.1(780-790)$ & - \\
\hline$\beta$-Caryophyllene oxide & $0.3(777-799)$ & - & - \\
\hline 11-Hexadecynal & - & - & $1.6(729-760)$ \\
\hline$\alpha$-Cadinol & $0.2(852-863)$ & - & $0.3(821-852)$ \\
\hline Neointermedeol & - & - & $1.3(828-861)$ \\
\hline$\beta$-Vetivol & $2.1(803-814)$ & $0.1(810-823)$ & $0.9(804-823)$ \\
\hline$\alpha$-Costol & - & - & $0.8(816-847)$ \\
\hline$\beta$-Isonootkatol & $0.2(813-826)$ & - & - \\
\hline Aristolene epoxide & - & - & $0.5(763-787)$ \\
\hline \multicolumn{2}{|c|}{ 8-Hydroxy-endo-Cycloisolongifoleene (770-781) } & - & - \\
\hline $\begin{array}{l}\text { Aromadendrene } \\
\text { oxide-(2) }\end{array}$ & - & - & $1.0(823-878)$ \\
\hline cis-9-Hexadecenal & - & - & $0.1(721-745)$ \\
\hline (Z)-9,17-Octadecadienal & - & - & $0.1(713-805)$ \\
\hline Anthracene & - & - & $0.2(908-953)$ \\
\hline Viridiflorene & - & - & $0.5(869-870)$ \\
\hline
\end{tabular}

Values are relative quantity (\%) (standard Index-reverse standard index). 
Table 2. Chemical composition of essential oil from P. guajava leaves.

\begin{tabular}{|c|c|c|c|}
\hline Compound Name & ive Quantity (\%) & Standard Index & Reverse Standard Index \\
\hline$\alpha$-Pinene & 25.5 & 834 & 836 \\
\hline$\Delta$-3-Carene & 8.8 & 787 & 788 \\
\hline$\beta$-Pinene & 0.5 & 902 & 912 \\
\hline Camphene & 0.2 & 871 & 875 \\
\hline trans-Isolimonene & 0.2 & 864 & 865 \\
\hline Terpinen-4-ol & 0.3 & 908 & 910 \\
\hline$\beta$-Fenchol & 0.5 & 830 & 849 \\
\hline L-Bornyl acetate & 2.2 & 929 & 929 \\
\hline trans-Pinocarvyl acetate & 0.2 & 818 & 851 \\
\hline Bornylene & 0.5 & 820 & 829 \\
\hline Bicycloelemene & 0.2 & 776 & 847 \\
\hline$\alpha$-Patchoulene & 0.3 & 778 & 796 \\
\hline Cedrene & 0.11 & 880 & 898 \\
\hline$\beta$-Chamigrene & 0.2 & 895 & 913 \\
\hline$\beta$-Himachalene & 0.3 & 901 & 910 \\
\hline Thujopsene-(I2) & 0.1 & 890 & 900 \\
\hline Cuparene & 2.6 & 892 & 894 \\
\hline (E)-Caryophyllene & 15.7 & 872 & 874 \\
\hline$\gamma$-Muurolene & 0.2 & 881 & 896 \\
\hline (E)-Nerolidol & 16.7 & 870 & 871 \\
\hline Aristolene epoxide & 0.7 & 757 & 803 \\
\hline Cedran-8-ol & 8.8 & 878 & 882 \\
\hline Widdrol & 0.6 & 747 & 750 \\
\hline Isospathulenol & 0.2 & 815 & 861 \\
\hline$\alpha$-Bisabolol & 2.1 & 834 & 885 \\
\hline Ledene oxide-(II) & 1.1 & 835 & 837 \\
\hline $\begin{array}{l}\text { 1,3,3-Trimethyl-2-(2-methyl- } \\
\text { cyclopropyl)-cyclohexene }\end{array}$ & 1.4 & 758 & 805 \\
\hline 8-Hydroxy-endo-Cycloisolongifolene & 0.3 & 812 & 836 \\
\hline Calarene epoxide & 0.1 & 753 & 814 \\
\hline Viridiflorene & 0.1 & 771 & 781 \\
\hline Labda-8(20),12,14-triene & 0.1 & 791 & 794 \\
\hline 13-Epimanool & 3.3 & 778 & 785 \\
\hline
\end{tabular}


Table 3. Chemical composition of essential oil from O. majorana aerial parts.

\begin{tabular}{|c|c|c|c|}
\hline Compound Name & Relative Quantity (\%) & Standard Index & Reverse Standard Index \\
\hline$\gamma$-Terpinene & 16.5 & 877 & 880 \\
\hline$\alpha$-Thujene & 3.2 & 915 & 922 \\
\hline Sabinene & 10.1 & 915 & 924 \\
\hline$\alpha$-Terpinene & 6.1 & 895 & 897 \\
\hline$\beta$-Thujene & 0.9 & 910 & 931 \\
\hline$\beta$-Phellandrene & 0.7 & 910 & 915 \\
\hline$p$-Cymene & 0.8 & 839 & 875 \\
\hline Terpinolene & 5.7 & 898 & 900 \\
\hline$\gamma$-Terpineol & 5.1 & 845 & 847 \\
\hline 4-Thujanol & 2.6 & 899 & 902 \\
\hline trans-4-Thujanol & 4.8 & 916 & 918 \\
\hline cis-Para-2-menthen-1-ol & 0.3 & 884 & 886 \\
\hline (E)-Caryophyllene & 2.5 & 894 & 895 \\
\hline Terpinen-4-ol & 21.7 & 876 & 882 \\
\hline$\alpha$-Terpineol & 5.1 & 914 & 925 \\
\hline trans-Piperitol & 0.1 & 833 & 865 \\
\hline$\gamma$-Terpinyl acetate & 6.7 & 830 & 830 \\
\hline Bornyl acetate & 0.3 & 911 & 945 \\
\hline Terpinyl propionate & 0.6 & 845 & 886 \\
\hline Neryl acetate & 0.5 & 891 & 897 \\
\hline$\gamma$-Elemene & 1.4 & 886 & 906 \\
\hline$\alpha$-Humulene & 0.1 & 863 & 880 \\
\hline Germacrene D & 1.6 & 892 & 894 \\
\hline Spathulenol & 0.5 & 902 & 903 \\
\hline Isospathulenol & 0.2 & 828 & 843 \\
\hline$\beta$-Caryophyllene oxide & 0.2 & 858 & 867 \\
\hline
\end{tabular}

\subsection{Red Flour Beetle Experiment}

\section{Fumigant Toxicity of Tested Essential Oils}

Figure 1 shows the statistical significance of the main effects (oil source, oil concentration, and time of exposure). Oil of S. terebinthifolius unripe fruits showed the highest mortality of T. castaneum (Figure 1A). With increased oil concentration and exposure time, mortality increased significantly (Figure 1B,C). Additionally, the interaction between two factors (Figure 1D-F) showed significant effects on the mortality percentage of T. castaneum. 

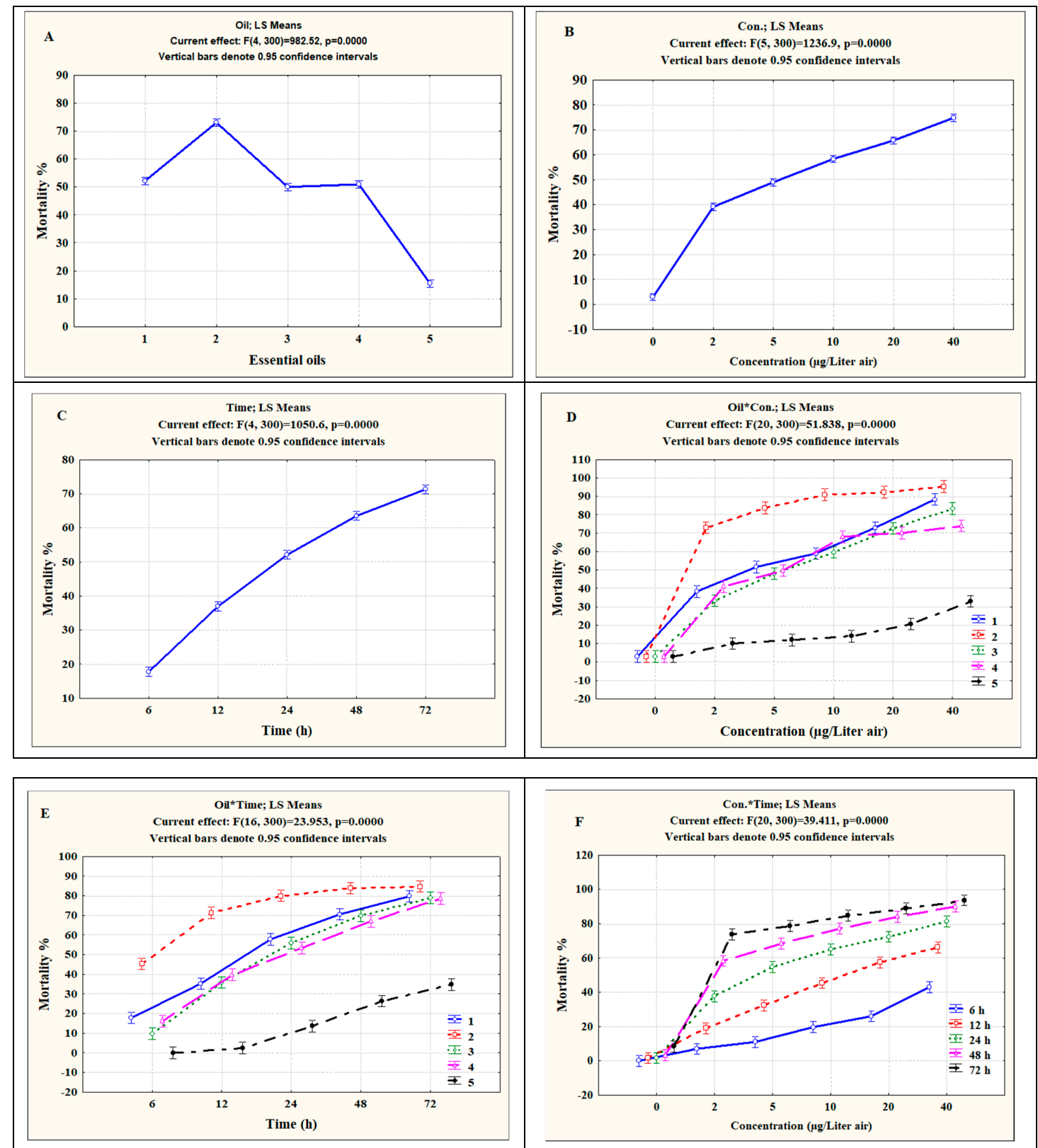

Figure 1. Effects among parameters of tested EOs on T. castaneum mortality: concentrations $(0,10,25,50$, and $100 \mu \mathrm{g} / \mathrm{L}$ air) and exposure time $(6,12,24$, and $72 \mathrm{~h}$ ). (A-C) Results of T. castaneum mortality when only one parameter effect was studied separately. (D-F) Results when effects between two parameters on T. castaneum mortality were studied together: (D) EO and concentration, (E) EO and exposure time, and (F) concentration and exposure time. 1: S. terebinthifolius ripe fruit oil. 2: S. terebinthifolius unripe fruit oil. 3: S. terebinthifolius leaf oil. 4: P. guajava leaf oil. 5: O. majorana air-dried aerial parts EO.

The mortality values were $31.66 \%$ and $75 \%$ after $24 \mathrm{~h}$ of exposure to EO of 0 . majorana leaves and P. guajava leaves, respectively, and were $56.66 \%$ and $93.33 \%$ after $48 \mathrm{~h}$. The mortality values were $68.33 \%$ and $100 \%$ after $72 \mathrm{~h}$ with EO O. majorana leaves and P. guajava leaves at $40 \mu \mathrm{g} / \mathrm{L}$ air, respectively (Table 4). The great effect of EO of S. terebinthifolius unripe fruits on adult T. castaneum after $6 \mathrm{~h}$ of exposure at $40 \mu \mathrm{g} / \mathrm{L}$ air was shown by adult mortality of $76.66 \%$. After $6 \mathrm{~h}$ at $40 \mu \mathrm{g} / \mathrm{L}$ air with EO of S. terebinthifolius leaves, O. majorana leaves, and P. guajava leaves, the adult mortality was under 50\%, while it was $61.66 \%$ and $76.66 \%$ with $\mathrm{EO}$ of $S$. terebinthifolius ripe and unripe fruits, respectively. 
Table 4. Mortality (\%) of Tribolium castaneum as affected by different concentrations of five essential oils with different time periods using a fumigant application.

\begin{tabular}{|c|c|c|c|c|c|c|}
\hline \multirow{2}{*}{$\begin{array}{c}\text { Source of } \\
\text { Essential Oil }\end{array}$} & \multirow{2}{*}{$\begin{array}{l}\text { Concentration } \\
(\mu \mathrm{g} / \mathrm{L} \text { Air })\end{array}$} & \multicolumn{5}{|c|}{ Time (h) } \\
\hline & & 6 & 12 & 24 & 48 & 72 \\
\hline \multirow{6}{*}{$\begin{array}{l}\text { S. terebinthifolius } \\
\text { ripe fruits }\end{array}$} & 0 & 0 & $1.6 \pm 2.8$ & $1.66 \pm 2.88$ & $3.3 \pm 2.8$ & $8.3 \pm 2.8$ \\
\hline & 2 & 0 & $16.6 \pm 14.4$ & $33.33 \pm 14.43$ & $58.3 \pm 7.6$ & $83.3 \pm 7.6$ \\
\hline & 5 & $6.6 \pm 5.7$ & $30 \pm 5$ & $58.33 \pm 7.63$ & $76.6 \pm 10.4$ & $86.6 \pm 5.7$ \\
\hline & 10 & $6.6 \pm 5.7$ & $33.3 \pm 2.8$ & $70.00 \pm 0.00$ & $85 \pm 5$ & 100 \\
\hline & 20 & $31.6 \pm 2.8$ & $50 \pm 5$ & $83.3 \pm 7.6$ & 100 & 100 \\
\hline & 40 & $61.6 \pm 12.5$ & $80 \pm 10$ & 100 & 100 & 100 \\
\hline \multirow{6}{*}{$\begin{array}{l}\text { S. terebinthifolius } \\
\text { unripe fruits }\end{array}$} & 0 & 0 & $1.6 \pm 2.8$ & $1.6 \pm 2.8$ & $3.3 \pm 2.8$ & $8.33 \pm 2.88$ \\
\hline & 2 & $35 \pm 5$ & $51.6 \pm 7.6$ & $78.3 \pm 7.6$ & 100 & 100 \\
\hline & 5 & $43.3 \pm 11.5$ & $75 \pm 5$ & 100 & 100 & 100 \\
\hline & 10 & $55 \pm 18$ & 100 & 100 & 100 & 100 \\
\hline & 20 & $61.6 \pm 12.5$ & 100 & 100 & 100 & 100 \\
\hline & 40 & $76.6 \pm 5.7$ & 100 & 100 & 100 & 100 \\
\hline \multirow{6}{*}{$\begin{array}{c}\text { S. terebinthifolius } \\
\text { leaves }\end{array}$} & 0 & 0 & $1.6 \pm 2.8$ & $1.6 \pm 2.8$ & $3.3 \pm 2.8$ & $8.3 \pm 2.8$ \\
\hline & 2 & 0 & $8.3 \pm 2.8$ & $28.3 \pm 5.7$ & $55 \pm 5$ & $75 \pm 10$ \\
\hline & 5 & 0 & $26.6 \pm 15.2$ & $50 \pm 5$ & $73.3 \pm 2.8$ & $90 \pm 5$ \\
\hline & 10 & $8.3 \pm 7.6$ & $33.3 \pm 7.6$ & $70 \pm 8.6$ & $86.6 \pm 5.7$ & 100 \\
\hline & 20 & 10 & $68.3 \pm 17.5$ & $85 \pm 10$ & 100 & 100 \\
\hline & 40 & $40 \pm 10$ & $76.6 \pm 5.7$ & 100 & 100 & 100 \\
\hline \multirow{6}{*}{ O. majorana leaves } & 0 & 0 & $1.66 \pm 2.8$ & $1.6 \pm 2.8$ & $3.3 \pm 2.8$ & $8.3 \pm 2.8$ \\
\hline & 2 & 0 & 0 & $6.6 \pm 2.8$ & $16.6 \pm 2.8$ & $26.6 \pm 2.8$ \\
\hline & 5 & 0 & 0 & 10 & $21.6 \pm 2.8$ & $28.3 \pm 2.8$ \\
\hline & 10 & 0 & 0 & $11.6 \pm 5.7$ & $26.6 \pm 2.8$ & $31.6 \pm 5.7$ \\
\hline & 20 & 0 & $5 \pm 5$ & $20 \pm 5$ & $33.3 \pm 5.7$ & $45 \pm 5$ \\
\hline & 40 & 0 & $8.3 \pm 2.8$ & $31.6 \pm 7.6$ & $56.6 \pm 15.2$ & $68.3 \pm 12.5$ \\
\hline \multirow{6}{*}{ P. guajava leaves } & 0 & 0 & $1.6 \pm 2.8$ & $1.6 \pm 2.8$ & $3.3 \pm 2.8$ & $8.3 \pm 2.8$ \\
\hline & 2 & 0 & $18.3 \pm 7.6$ & $41.6 \pm 7.6$ & $61.6 \pm 5.7$ & $83.3 \pm 12.5$ \\
\hline & 5 & $5 \pm 5$ & $30 \pm 5$ & $55 \pm 5$ & $70 \pm 5$ & $88.3 \pm 12.5$ \\
\hline & 10 & $28.3 \pm 7.6$ & $60 \pm 10$ & $73.3 \pm 7.6$ & $86.6 \pm 5.7$ & $91.6 \pm 14.4$ \\
\hline & 20 & $26.6 \pm 2.8$ & $63.3 \pm 12.5$ & $73.3 \pm 7.6$ & $86.6 \pm 5.7$ & 100 \\
\hline & 40 & $36.6 \pm 5.7$ & $65 \pm 13.2$ & $75 \pm 13.2$ & $93.3 \pm 11.5$ & 100 \\
\hline \multicolumn{7}{|c|}{$\mathrm{LSD}_{0.05}^{*}=10.077$} \\
\hline
\end{tabular}

* LSD: Least Significant Difference

After $72 \mathrm{~h}$ at $5 \mu \mathrm{g} / \mathrm{L}$ air with EOs of S. terebinthifolius ripe fruits, unripe fruits, and leaves, O. majorana leaves, and P. guajava leaves, adult mortality was $86.66 \%, 100 \%, 90 \%, 28.33 \%$, and $88.33 \%$, respectively, while the control was recorded as a standard reference. After 12 and $24 \mathrm{~h}$ of exposure with acetone as the control, mortality was $1.66 \%$, but was $3.33 \%$ after $48 \mathrm{~h}$ and $8.33 \%$ after $72 \mathrm{~h}$ (Table 4 ).

The fumigant experiment applied to adult T. castaneum with different times and concentrations showed that adult mortality increased gradually with increased concentrations from 2 to $40 \mu \mathrm{g} / \mathrm{L}$ air and time from $6 \mathrm{~h}$ to $72 \mathrm{~h}$ of exposure.

Figure 2 illustrates the effects of tested EOs on adult T. castaneum, with dead insects (shown in black) due to accumulation of $\mathrm{CO}_{2}$ in the tracheas of insects treated with the fumigation method, when compared to normal T. castaneum (in brown). 


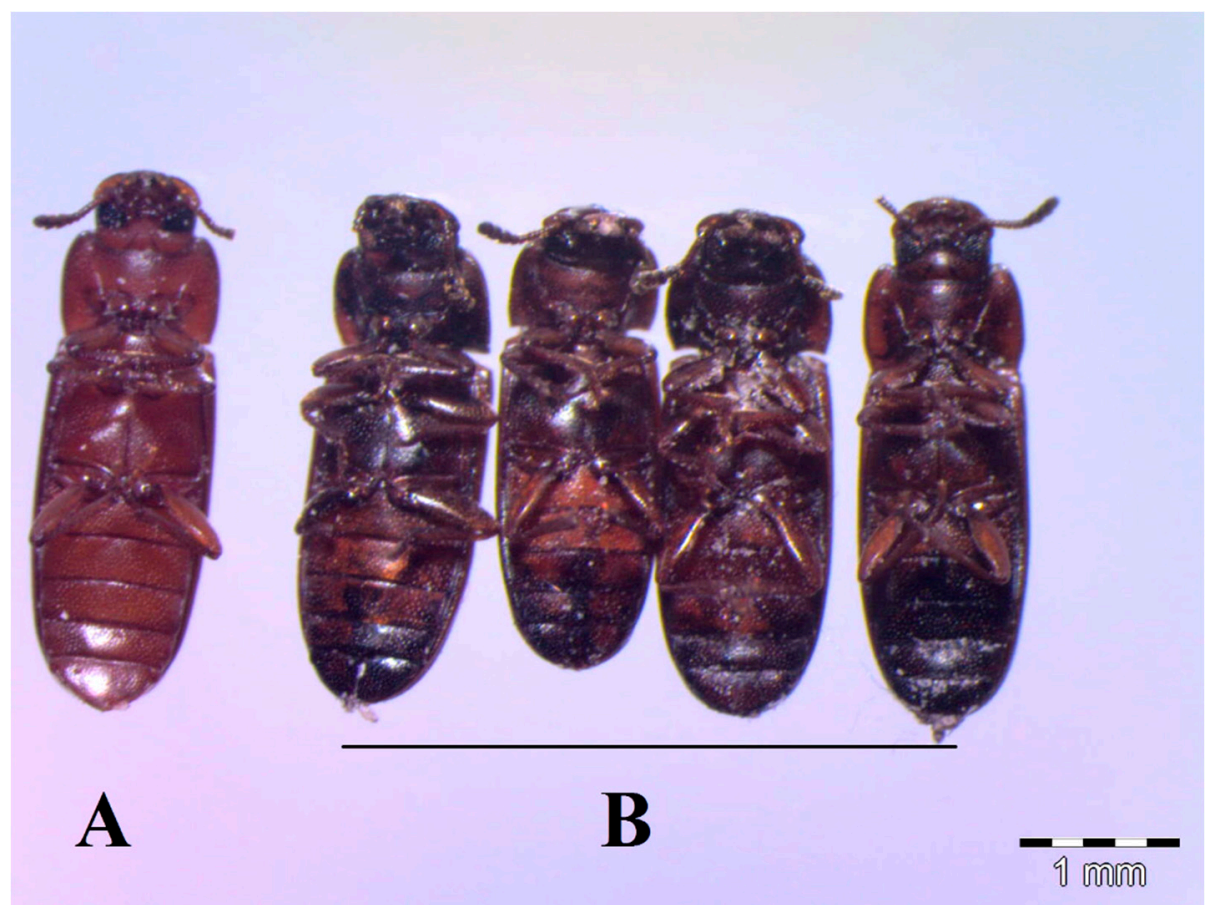

Figure 2. (A) Adult T. castaneum from the control group. (B) Adult mortality of T. castaneum after treatment with $\mathrm{S}$. terebinthifolius unripe fruit $\mathrm{EO}$ at higher concentrations using the fumigant method with different time periods.

Table 3 presents the mortality percentages of $T$. castaneum as affected by the three factors, oil source, oil concentration, and time period, with fumigant application. After $48 \mathrm{~h}$ of treatment, the mortality ranged from $58.3 \%$ to $100 \%$ with EO of S. terebinthifolius ripe fruits, was $100 \%$ with EO of S. terebinthifolius unripe fruits, ranged from $55 \%$ to $100 \%$ with $\mathrm{EO}$ of S. terebinthifolius leaves, and ranged from $16.6 \%$ to $56.6 \%$ with EO of O. majorana leaves, and $61.6 \%$ to $93.3 \%$ with EO of P. guajava leaves. By comparison, mortality was $8.3 \%$ with the control (Table 2).

The lethal concentration causing $50 \%$ mortality $\left(\mathrm{LC}_{50}\right)$ of $T$. castaneum was calculated for tested EOs at different time periods $(6,12,24,48$, and $72 \mathrm{~h})$. After $6 \mathrm{~h}$ of treatment, the $\mathrm{LC}_{50}$ was 33.3, $6.8,>40,>40$, and $>40 \mu \mathrm{g} / \mathrm{L}$ air for EOs of $S$. terebinthifolius ripe fruits, S. terebinthifolius unripe fruits, S. terebinthifolius leaves, O. majorana leaves, and P. guajava leaves, respectively. After $24 \mathrm{~h}$ of treatment, the $\mathrm{LC}_{50}$ was $4.2,<2,5.1,>40$, and $6.1 \mu \mathrm{g} / \mathrm{L}$ air for EOs of $S$. terebinthifolius ripe fruits, $S$. terebinthifolius leaves, O. majorana leaves, and P. guajava leaves, respectively. After $24 \mathrm{~h}$ of treatment, the $\mathrm{LC}_{50}$ of S. terebinthifolius unripe fruit oil was under $2 \mu \mathrm{g} / \mathrm{L}$ air, which means that the EO of $S$. terebinthifolius ripe fruits had a stronger effect on T. castaneum adults than other tested EOs using the fumigation method (Table 5). 
Table 5. Probit regression line parameters of T. castaneum for five essential oils at five interval concentrations.

\begin{tabular}{|c|c|c|c|c|c|c|}
\hline \multirow{2}{*}{ Tested Essential Oil } & \multirow{2}{*}{ Period (h) } & \multirow{2}{*}{$\mathrm{LC}_{50}(\mu \mathrm{g} / \mathrm{L} \mathrm{Air})$} & \multicolumn{2}{|c|}{ 95\% Confidence Limits } & \multirow{2}{*}{ Slope $\pm \mathrm{SE}^{*}$} & \multirow{2}{*}{$\mathrm{Chi}^{2}$} \\
\hline & & & Lower & Upper & & \\
\hline \multirow{5}{*}{$\begin{array}{l}\text { S. terebinthifolius ripe } \\
\text { fruits }\end{array}$} & 6 & 33.3 & 20.3 & 54.6 & $2.1 \pm 0.1$ & 0.59 \\
\hline & 12 & 15.5 & 8 & 30.2 & $1.2 \pm 0.1$ & 0.92 \\
\hline & 24 & 4.2 & 2.2 & 8.1 & $1.3 \pm 0.1$ & 0.98 \\
\hline & 48 & $<2$ & & & & \\
\hline & 72 & $<2$ & & & & \\
\hline \multirow{5}{*}{$\begin{array}{l}\text { S. terebinthifolius } \\
\text { unripe fruits }\end{array}$} & 6 & 6.8 & 2.5 & 18.4 & $0.8 \pm 0.2$ & 0.99 \\
\hline & 12 & 2 & 1.1 & 3.8 & $1.5 \pm 0.1$ & NA \\
\hline & 24 & $<2$ & & & & \\
\hline & 48 & $<2$ & & & & \\
\hline & 72 & $<2$ & & & & \\
\hline \multirow{5}{*}{$\begin{array}{l}\text { S. terebinthifolius } \\
\text { leaves }\end{array}$} & 6 & $>40$ & & & & \\
\hline & 12 & 14.5 & 8.5 & 24.5 & $1.7 \pm 0.1$ & 0.95 \\
\hline & 24 & 5.1 & 2.8 & 8.8 & $1.5 \pm 0.1$ & 0.99 \\
\hline & 48 & $<2$ & & & & \\
\hline & 72 & $<2$ & & & & \\
\hline \multirow{5}{*}{ O. majorana leaves } & 6 & $>40$ & & & & \\
\hline & 12 & $>40$ & & & & \\
\hline & 24 & $>40$ & & & & \\
\hline & 48 & $>40$ & & & & \\
\hline & 72 & 37.9 & 13.9 & 103.1 & $0.8 \pm 0.2$ & 0.85 \\
\hline \multirow{5}{*}{ P. guajava leaves } & 6 & $>40$ & & & & \\
\hline & 12 & 9.5 & 4.5 & 16.4 & $1.4 \pm 0.3$ & 0.93 \\
\hline & 24 & 6.1 & 1.8 & 19.7 & $0.6 \pm 0.2$ & 0.97 \\
\hline & 48 & $<2$ & & & & \\
\hline & 72 & $<2$ & & & & \\
\hline
\end{tabular}

\subsection{Insecticidal Activity of Essential Oil on C. Pipiens}

\subsubsection{Immature Stages}

Table 6 shows the significant effects of oil concentrations and oil sources and their interaction with mortality and longevity of C. pipiens at different stages (larval, pupal, and adult). All treatments showed highly significant effects on mortality and longevity except the interaction between the EO source and the EO concentration for longevity at the larval stage. 
Table 6. Analysis of variance for the effect of main treatments and their combinations on C. pipiens.

\begin{tabular}{|c|c|c|c|c|c|}
\hline S.O.V.* & DF & Sum of Squares & Mean Square & F-test Value & $\operatorname{Pr}>F$ \\
\hline Larval stage & \multicolumn{5}{|c|}{ Mortality after $24 \mathrm{~h}(\%)$} \\
\hline $\begin{array}{c}\text { Oil } \\
\text { concentration } \\
\text { (A) }\end{array}$ & 4 & 6235.73 & 1558.93 & 186.18 & $<0.0001$ \\
\hline Oil source $(B)$ & 4 & 1504 & 376 & 44.90 & $<0.0001$ \\
\hline$A \times B$ & 16 & 988.26 & 61.76 & 7.38 & $<0.0001$ \\
\hline Error & 50 & 418.66 & 8.37 & & \\
\hline \multicolumn{6}{|c|}{ Mortality after 48 h (\%) } \\
\hline A & 4 & 7212.58 & 1803.146 & 193.19 & $<0.0001$ \\
\hline B & 4 & 1546.98 & 386.74 & 41.44 & $<0.0001$ \\
\hline$A \times B$ & 16 & 959.14 & 59.94 & 6.42 & $<0.0001$ \\
\hline Error & 50 & 466.66 & 9.33 & & \\
\hline \multicolumn{6}{|c|}{ Total mortality (\%) } \\
\hline A & 4 & $22,063.14$ & 5515.78 & 415.35 & $<0.0001$ \\
\hline B & 4 & 7092.48 & 1773.12 & 133.52 & $<0.0001$ \\
\hline$A \times B$ & 16 & 2720.85 & 170.053 & 12.81 & $<0.0001$ \\
\hline Error & 50 & 664 & 13.28 & & \\
\hline \multicolumn{6}{|c|}{ Longevity (days) } \\
\hline $\mathrm{A}$ & 4 & 992.89 & 48.22 & 30.94 & $<0.0001$ \\
\hline B & 4 & 167.718 & 41.929 & 5.23 & 0.0013 \\
\hline $\mathrm{A} \times \mathrm{B}$ & 16 & 78.8658 & 4.9291 & 0.61 & 0.8571 \\
\hline Error & 50 & 401.153 & 8.023 & & \\
\hline Pupal stage & \multicolumn{5}{|c|}{ Mortality (\%) } \\
\hline $\mathrm{A}$ & 4 & $26,178.66$ & 6544.66 & 475.63 & $<0.0001$ \\
\hline B & 4 & 7129.06 & 1782.26 & 129.53 & $<0.0001$ \\
\hline$A \times B$ & 16 & 2540.26 & 158.76 & 11.54 & $<0.0001$ \\
\hline \multirow[t]{2}{*}{ Error } & 50 & 688. & 13.76 & & \\
\hline & \multicolumn{5}{|c|}{ Longevity (h) } \\
\hline $\mathrm{A}$ & 4 & 2292.65 & 573.16 & 17.31 & $<0.0001$ \\
\hline B & 4 & 2734.14 & 683.53 & 20.65 & $<0.0001$ \\
\hline$A \times B$ & 16 & 1040.83 & 65.05 & 1.96 & 0.0355 \\
\hline Error & 50 & 1655.27 & 33.11 & & \\
\hline Adult stage & \multicolumn{5}{|c|}{ Mortality (\%) } \\
\hline $\mathrm{A}$ & 4 & $47,423.78$ & $11,855.94$ & 835.71 & $<0.0001$ \\
\hline B & 4 & 9540.05 & 2385.01 & 168.12 & $<0.0001$ \\
\hline $\mathrm{A} \times \mathrm{B}$ & 16 & 2717.54 & 169.84 & 11.97 & $<0.0001$ \\
\hline Error & 50 & 709.33 & 14.18 & & \\
\hline \multicolumn{6}{|c|}{ Longevity (days) } \\
\hline $\mathrm{A}$ & 4 & 6651.02 & 1662.75 & 94.69 & $<0.0001$ \\
\hline B & 4 & 1961.75 & 490.43 & 27.93 & $<0.0001$ \\
\hline $\mathrm{A} \times \mathrm{B}$ & 16 & 767.807 & 47.98 & 2.73 & 0.0034 \\
\hline Error & 50 & 878.04 & 17.56 & & \\
\hline
\end{tabular}


The EOs were tested for their toxicity against the second instar larvae of $C$. pipiens. The five EOs showed pronounced insecticidal activity on immature stages (larva and pupa). After $24 \mathrm{~h}$ of treatment with EO of $S$. terebinthifolius ripe fruits, unripe fruits, and leaves, O. majorana leaves, and $P$. guajava leaves, the larval mortality was $15.3 \%, 34.6 \%, 30.6 \%, 36.6 \%$, and $16.6 \%$ at $100 \mathrm{mg} / \mathrm{L}$, respectively (Table 7). The larval mortality recorded after $48 \mathrm{~h}$ of treatment with the tested EOs was $17.3 \%, 36.6 \%$, $32.6 \%, 38.6 \%$, and $18.6 \%$ at $100 \mathrm{mg} / \mathrm{L}$, respectively.

The total larval mortality was recorded during the larval stage for each concentration to examine the larvicidal activity of the tested EOs against C. pipiens. Table 7 shows that total larval mortality ranged from $26 \%$ to $33.3 \%$ with EO of S. terebinthifolius ripe fruits, $40.6 \%$ to $68 \%$ with EO of S. terebinthifolius unripe fruits, $30 \%$ to $50 \%$ with EO of S. terebinthifolius leaves, $42.6 \%$ to $78 \%$ with EO of O. majorana leaves, and $24 \%$ to $36.6 \%$ with EO of P. guajava leaves at 10 to $100 \mathrm{mg} / \mathrm{L}$, and was $3.3 \%$ as a control. Mortality increased with growing concentration and time of exposure.

The present data confirm that the EOs of O. majorana leaves and S. terebinthifolius unripe fruits and leaves were more effective as larvicide than EOs of $S$. terebinthifolius ripe fruits and $P$. guajava leaves on C. pipiens at a higher concentration $(100 \mathrm{mg} / \mathrm{L})$.

Figures 3 and 4 show the destroyed digestive system (rupture) in larvae of $C$. pipiens, which results in increased larval mortality within a short time (24-48 h) with treatment by EO of S. terebinthifolius unripe fruits, while EO of O. majorana leaves led to a $78 \%$ mortality at $100 \mathrm{mg} / \mathrm{L}$.

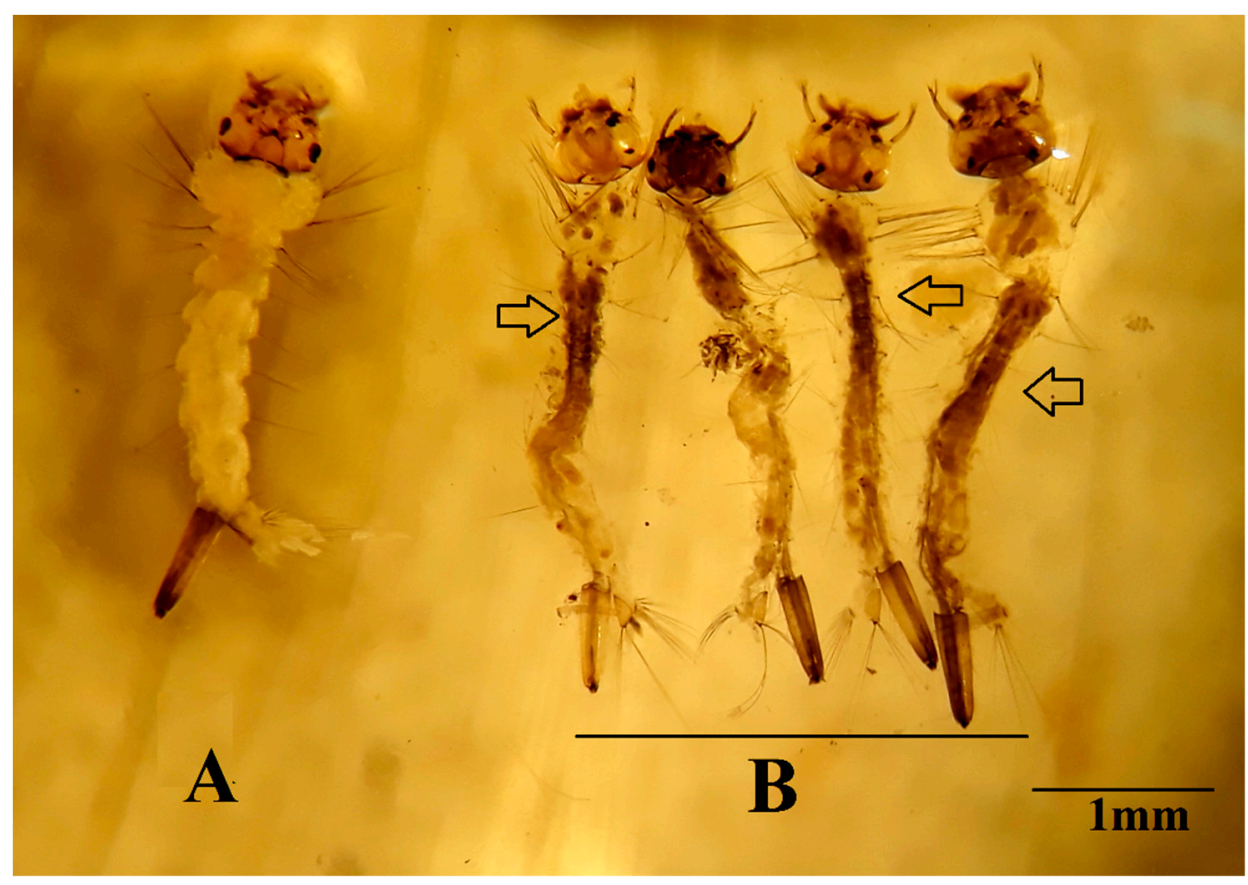

Figure 3. (A) Normal C. pipiens larva from the control. (B) Abnormal larvae produced after treatment with oil of S. terebinthifolius unripe fruits at $100 \mathrm{mg} / \mathrm{L}$ showing a destroyed digestive system, especially midgut (arrows). 
Table 7. Insecticidal effect of tested essential oils on biological activity of C. pipiens.

\begin{tabular}{|c|c|c|c|c|c|c|c|c|c|}
\hline \multirow{2}{*}{$\begin{array}{c}\text { Tested } \\
\text { Essential Oil }\end{array}$} & \multirow{2}{*}{$\begin{array}{c}\text { Concentration } \\
(\mathrm{mg} / \mathrm{L})\end{array}$} & \multicolumn{4}{|c|}{ Larval Stage } & \multicolumn{2}{|c|}{ Pupal Stage } & \multicolumn{2}{|c|}{ Adult Stage } \\
\hline & & $\begin{array}{c}\text { Mortality } \\
\text { after } 24 \text { h (\%) }\end{array}$ & $\begin{array}{c}\text { Mortality } \\
\text { after } 48 \text { h (\%) }\end{array}$ & $\begin{array}{c}\text { Total } \\
\text { Mortality (\%) }\end{array}$ & Longevity (days) & Mortality (\%) & Longevity (h) & Mortality (\%) & Longevity (days) \\
\hline \multirow{5}{*}{$\begin{array}{l}\text { S. terebinthifolius } \\
\text { ripe fruits }\end{array}$} & 0 & $0.6 \pm 1.1$ & $0.6 \pm 1.1$ & $3.3 \pm 1.1$ & $8.3 \pm 0.6$ & $4 \pm 2$ & $32.2 \pm 6.7$ & $5.3 \pm 1.1$ & $44.3 \pm 6.1$ \\
\hline & 10 & $10 \pm 2$ & $10 \pm 2$ & $26 \pm 2$ & $11.2 \pm 0.8$ & $26 \pm 2$ & $36.7 \pm 10.5$ & $36 \pm 2$ & $44.6 \pm 2.1$ \\
\hline & 25 & $10.6 \pm 3$ & $11.3 \pm 2.3$ & $26.6 \pm 3$ & $14.4 \pm 2.9$ & $26.6 \pm 3$ & $46.1 \pm 12.4$ & $38 \pm 5.2$ & $40.6 \pm 3$ \\
\hline & 50 & $12 \pm 2$ & $13.3 \pm 2.3$ & $30.6 \pm 3$ & $18.5 \pm 3.4$ & $32.6 \pm 3$ & $57 \pm 4.4$ & $49.3 \pm 3$ & $32 \pm 6.4$ \\
\hline & 100 & $15.3 \pm 1.1$ & $17.3 \pm 1.1$ & $33.3 \pm 3$ & $20.6 \pm 2.1$ & $40 \pm 4$ & $63.4 \pm 1.6$ & $58 \pm 4$ & $27.3 \pm 3.2$ \\
\hline \multirow{5}{*}{$\begin{array}{l}\text { S. terebinthifolius } \\
\text { unripe fruits }\end{array}$} & 0 & $0.6 \pm 1.1$ & $0.6 \pm 1.1$ & $3.3 \pm 1.1$ & $8.3 \pm 0.6$ & $4 \pm 2$ & $32.2 \pm 6.7$ & $5.3 \pm 1.1$ & $44.3 \pm 6.1$ \\
\hline & 10 & $13.3 \pm 4.1$ & $14 \pm 4$ & $40.6 \pm 9.4$ & $9.4 \pm 1.8$ & $42.6 \pm 9.4$ & $25.1 \pm 6.5$ & $58.6 \pm 9.4$ & $33.7 \pm 4.7$ \\
\hline & 25 & $18 \pm 2$ & $18.6 \pm 1.1$ & $48 \pm 2$ & $10.5 \pm 2.4$ & $45.3 \pm 5.7$ & $29.8 \pm 4.7$ & $63.3 \pm 5.7$ & $22.6 \pm 4$ \\
\hline & 50 & $28 \pm 2$ & $29.3 \pm 2.3$ & $60 \pm 2$ & $13.7 \pm 3.2$ & $64 \pm 2$ & $34.4 \pm 2.5$ & $84 \pm 2$ & $18.1 \pm 1.6$ \\
\hline & 100 & $34.6 \pm 5.7$ & $36.6 \pm 5.7$ & $68 \pm 3.4$ & $16.3 \pm 0.9$ & $72 \pm 3.4$ & $37.1 \pm 6.3$ & $94 \pm 6$ & $15.4 \pm 4.4$ \\
\hline \multirow{4}{*}{$\begin{array}{l}\text { S. terebinthifolius } \\
\text { leaves }\end{array}$} & 0 & $0.6 \pm 1.1$ & $0.6 \pm 1.1$ & $3.3 \pm 1.1$ & $8.3 \pm 0.6$ & $4 \pm 2$ & $32.2 \pm 6.7$ & $5.3 \pm 1.1$ & $44.3 \pm 6.1$ \\
\hline & 10 & $9.3 \pm 1.1$ & $10 \pm 2$ & $30 \pm 2$ & $8.5 \pm 5.1$ & $30 \pm 2$ & $33.3 \pm 3.9$ & $46 \pm 2$ & $34.4 \pm 7.3$ \\
\hline & 50 & $27.3 \pm 4.1$ & $28.6 \pm 5$ & $48.6 \pm 5$ & $14.7 \pm 5.5$ & $52.6 \pm 5$ & $38.5 \pm 4.9$ & $70.6 \pm 5$ & $18.8 \pm 2.2$ \\
\hline & 100 & $30.6 \pm 5$ & $32.6 \pm 5$ & $50 \pm 2$ & $18.2 \pm 2.4$ & $58 \pm 2$ & $43.1 \pm 6.4$ & $78 \pm 2$ & $20.5 \pm 3.2$ \\
\hline \multirow{5}{*}{$\begin{array}{l}\text { O. majorana } \\
\text { leaves }\end{array}$} & 0 & $0.6 \pm 1.1$ & $0.6 \pm 1.1$ & $3.3 \pm 1.1$ & $8.3 \pm 0.6$ & $4 \pm 2$ & $32.2 \pm 6.7$ & $5.3 \pm 1.1$ & $44.3 \pm 6.1$ \\
\hline & 10 & $12 \pm 5.2$ & $12.6 \pm 6.4$ & $42.6 \pm 6.4$ & $8.1 \pm 2.7$ & $44.6 \pm 6.4$ & $22.1 \pm 4.1$ & $62.6 \pm 6.4$ & $30.4 \pm 2$ \\
\hline & 25 & $19.3 \pm 1.1$ & $20 \pm 2$ & $50 \pm 2$ & $9.3 \pm 2.4$ & $52 \pm 2$ & $28.8 \pm 3$ & $70 \pm 2$ & $19.4 \pm 1.2$ \\
\hline & 50 & $30.6 \pm 3$ & $32 \pm 4$ & $62 \pm 4$ & $12.3 \pm 4.9$ & $66 \pm 4$ & $30.7 \pm 5.1$ & $86 \pm 4$ & $14.8 \pm 1.4$ \\
\hline & 100 & $36.6 \pm 4.1$ & $38.6 \pm 4.1$ & $78 \pm 2$ & $14.8 \pm 2.8$ & $82 \pm 2$ & $34.7 \pm 3.7$ & 100 & $11.8 \pm 2.4$ \\
\hline \multirow{5}{*}{$\begin{array}{c}\text { Psidium } \\
\text { guajava leaves }\end{array}$} & 0 & $0.6 \pm 1.1$ & $0.6 \pm 1.1$ & $3.3 \pm 1.1$ & $8.3 \pm 0.6$ & $4 \pm 2$ & $32.2 \pm 6.7$ & $5.3 \pm 1.1$ & $44.3 \pm 6.1$ \\
\hline & 10 & $8 \pm 2$ & $8 \pm 2$ & $24 \pm 2$ & $9.5 \pm 1.5$ & $24 \pm 2$ & $31.6 \pm 3.4$ & $34.6 \pm 2.3$ & $45 \pm 3$ \\
\hline & 25 & $9.3 \pm 1.1$ & 10 & 26 & $13.7 \pm 3.1$ & $27.3 \pm 2.3$ & $35.2 \pm 3.6$ & $37.3 \pm 2.3$ & $38.6 \pm 1.1$ \\
\hline & 50 & $12.6 \pm 3$ & $14 \pm 3.4$ & $30 \pm 3.4$ & $17.4 \pm 1.9$ & $33.3 \pm 3$ & $41.7 \pm 3.4$ & $51.3 \pm 3$ & $21.7 \pm 4.3$ \\
\hline & 100 & $16.6 \pm 1.1$ & $18.6 \pm 1.1$ & $36.6 \pm 8.1$ & $19.9 \pm 2.6$ & $42.6 \pm 5$ & $46.4 \pm 3.6$ & $63.3 \pm 3$ & $24.9 \pm 0.8$ \\
\hline \multicolumn{2}{|c|}{ P-value } & 0.0002 & $<0.0001$ & $<0.0001$ & 0.8571 & $<0.0001$ & 0.0355 & $<0.0001$ & 0.0034 \\
\hline
\end{tabular}




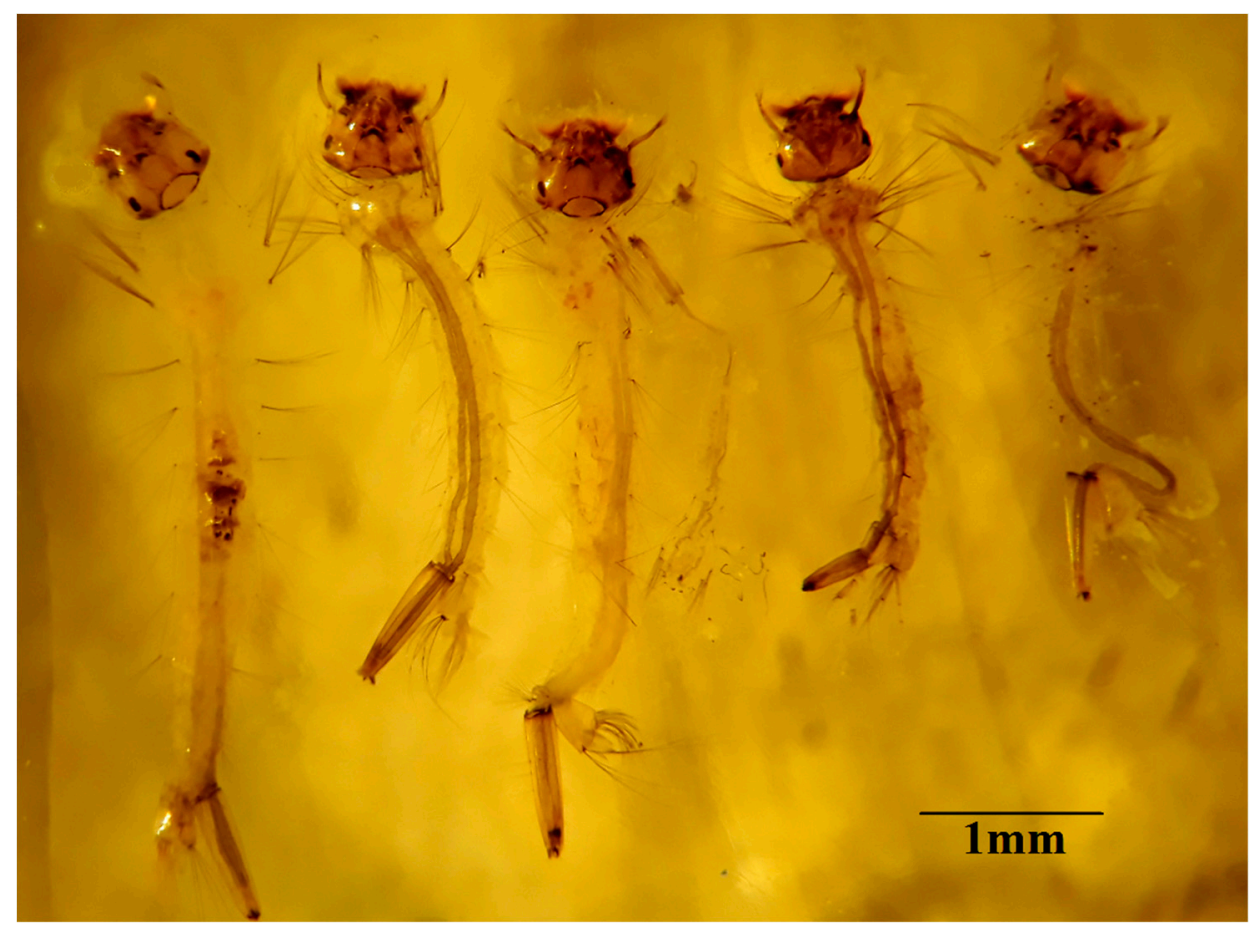

Figure 4. Abnormal larvae produced after treatment with $O$. majorana leaf oil at $100 \mathrm{mg} / \mathrm{L}$ showing a destroyed digestive system leading to a transparent midgut.

The effects of the tested EOs on immature stages were recorded as mortality percentages. As shown in Table 3, the mortality percentages increased gradually with increased oil concentration (from 10 to $100 \mathrm{mg} / \mathrm{L}$ ). Pupal mortality ranged from $26 \%$ to $40 \%$ with EO of $S$. terebinthifolius ripe fruits, $42.6 \%$ to $72 \%$ with EO of S. terebinthifolius unripe fruits, $30 \%$ to $58 \%$ with EO of S. terebinthifolius leaves, $44.6 \%$ to $82 \%$ with EO of O. majorana leaves, and $24 \%$ to $42.6 \%$ with EO of P. guajava leaves at 10 to $100 \mathrm{mg} / \mathrm{L}$. The tested EO of S. terebinthifolius ripe fruits, S. terebinthifolius unripe fruits, S. terebinthifolius leaves, $O$. majorana leaves, and P. guajava leaves affected larval and pupal longevity of $C$. pipiens. Larval longevity at $100 \mathrm{mg} / \mathrm{L}$ was 20.6, 16.3,18.2, 14.8, and 19.9 days, respectively, while it was 8.3 days in the control (Table 7).

On the other hand, pupal longevity was affected by treatment with $100 \mathrm{mg} / \mathrm{L}$ of EO of S. terebinthifolius ripe or unripe fruits and leaves, O. majorana leaves, and P. guajava leaves, with values at 63.4, 37.1, 43.1, and $46.4 \mathrm{~h}$, respectively, while it was $32.2 \mathrm{~h}$ in the control (Table 4).

\subsubsection{Adult Stage}

Adult mortality ranged from $36 \%$ to $58 \%$ with EO of S. terebinthifolius ripe fruits, $58.6 \%$ to $94 \%$ with EO of S. terebinthifolius unripe fruits, $46 \%$ to $78 \%$ with EO of S. terebinthifolius leaves, $62.6 \%$ to $100 \%$ with EO of O. majorana leaves, and $34.6 \%$ to $63.3 \%$ with EO of $P$. guajava leaves at $100 \mathrm{mg} / \mathrm{L}$, and was $5.3 \%$ in the control. Mortality increased with a growing concentration and time of exposure (Table 7).

Adult longevity reached 27.3, 15.4, 20.5, 11.8, and 24.9 days with $100 \mathrm{mg} / \mathrm{L}$ of EO of S. terebinthifolius ripe fruits, unripe fruits, and leaves, O. majorana leaves, and P. guajava leaves, respectively, and was 44.3 days with the control. EO from O. majorana leaves and S. terebinthifolius unripe fruits strongly reduced adult longevity by approximately $65 \%$ to $73 \%$ when compared with the control, which means that both EOs had insecticidal activity on the adult stage, which is an important vector for severe and highly infectious diseases in humans. 


\subsection{Lethal Concentrations of $L C_{50}$}

The results were obtained using probit regression line parameters of $C$. pipiens with five essential oils at five interval concentrations, and the lethal concentration causing $50 \%$ mortality $\left(\mathrm{LC}_{50}\right)$ was calculated for the tested EOs on larval and adult stages at different time periods (after 6, 12, 24, 48, and $72 \mathrm{~h}$ ) to examine the larvicidal and insecticidal activity.

The $\mathrm{LC}_{50}$ values of total larval mortality were $>100,31.2,>100,24.1$, and $>100 \mathrm{mg} / \mathrm{L}$ for EOs of S. terebinthifolius ripe fruits, unripe fruits, and leaves, O. majorana leaves, and P. guajava leaves (Table 8), respectively. This means that the oils of O. majorana leaves and S. terebinthifolius unripe fruits had stronger larvicidal activity against $C$. pipiens larvae than the other tested EOs applied by the dipping method.

Table 8. Probit regression line parameters of Culex pipiens for five essential oils at five interval concentrations.

\begin{tabular}{|c|c|c|c|c|c|c|c|}
\hline \multirow{2}{*}{ Oil Source } & \multirow{2}{*}{$\begin{array}{c}\text { Insect } \\
\text { Mortality }\end{array}$} & \multirow{2}{*}{$\begin{array}{c}\mathrm{LC}_{50} \\
(\mathrm{mg} / \mathrm{L})\end{array}$} & \multicolumn{2}{|c|}{ 95\% Confidence Limits } & \multirow{2}{*}{ Slope \pm SE } & \multirow{2}{*}{$\mathrm{Chi}^{2}$} & \multirow{2}{*}{$\mathbf{R}^{2}$} \\
\hline & & & Lower & Upper & & & \\
\hline \multirow{2}{*}{$\begin{array}{l}\text { S. terebinthifolius } \\
\text { ripe fruits }\end{array}$} & $\mathrm{TL}^{*}$ & $>100$ & - & - & - & - & - \\
\hline & $\mathrm{Ad}^{*}$ & $>50$ & - & - & - & - & - \\
\hline \multirow{2}{*}{$\begin{array}{l}\text { S. terebinthifolius } \\
\text { unripe fruits }\end{array}$} & $\mathrm{TL}$ & 31.2 & 8.8 & 109.6 & $0.721 \pm 0.2$ & 0.9 & 0.9 \\
\hline & Ad & 10.9 & 4.7 & 24.9 & $1.185 \pm 0.2$ & 0.8 & 0.9 \\
\hline \multirow{2}{*}{ S. terebinthifolius leaf } & $\mathrm{TL}$ & $>100$ & - & - & - & - & - \\
\hline & Ad & 20.1 & 6.9 & 57.3 & $0.872 \pm 0.2$ & 0.9 & 0.9 \\
\hline \multirow{2}{*}{ O. majorana leaf } & $\mathrm{TL}$ & 24.1 & 8.9 & 64.7 & $0.925 \pm 0.2$ & 0.9 & 0.9 \\
\hline & Ad & 9.7 & 4.6 & 20.1 & $1.414 \pm 0.1$ & 0.8 & 0.9 \\
\hline \multirow{2}{*}{ P. guajava leaf } & $\mathrm{TL}$ & $>100$ & - & - & - & - & - \\
\hline & Ad & $>50$ & - & - & - & - & - \\
\hline
\end{tabular}

In addition, Table 8 shows that the $\mathrm{LC}_{50}$ of adults was $>50,10.9,20.1,9.7$, and $>50 \mathrm{mg} / \mathrm{L}$ for EOs of $S$. terebinthifolius ripe fruits, unripe fruits, and leaves, O. majorana leaves, and P. guajava leaves, respectively. Therefore, the essential oils of $O$. majorana leaves and $S$. terebinthifolius unripe fruits had strong insecticidal activity against $C$. pipiens.

\section{Discussion}

\subsection{Chemical Constituents of the Essential Oils}

Several compounds have been identified in the studied plant materials. $\alpha$-Pinene was identified with a high percentage in EO from unripe fruits of $S$. terebinthifolius, which agreed with Ennigrou et al. [21], who reported that $\alpha$-pinene was found in amounts of $26.3 \%$ (immature fruits) and $13.9 \%$ (mature fruits). $\alpha$-Cadinol, elemol, germacrene-D, and $\Delta-3$-carene are the most common compounds identified in the EO of leaves and fruits of S. terebinthifolius [53]. $\Delta$-3-carene (25.9\%) was the most abundant compound in EO of $S$. terebinthifolius ripe fruits. Previously it was reported that the main chemical compounds of EO from S. terebinthifolius ripe fruits from Brazil were myrcene, limonene, and germacrene-D [54], while, in another report, $\Delta$-3-carene, and $\alpha$-pinene dominated in fruit EO [23].

$\Delta$-3-Carene, limonene, $\alpha$-phellandrene, and $\alpha$-pinene were reported as the major components of the EO of S. terebinthifolius fruits grown in Brazil [55]. Limonene, $\alpha$-phellandrene, $\alpha$-pinene, and germacrene-D were identified as the main compound of fruit essential oils of $S$. terebinthifolius from Reunion Island [56]. S. terebinthifolius fruit EOs in Germany showed $\alpha$-apinene, $\alpha$-phellandrene, $\beta$-phellandrene, and limonene [57], $\alpha$-phellandrene, $\gamma$-cadinene, $\beta$-phellandrene, $p$-cymene, and 
$\alpha$-pinene from Sfax (Southern Tunisia) [58], and from S. terebinthifolius ripe fruits in Egypt were $\alpha$-pinene, $\alpha$-phellandrene, limonene, $\alpha$-terpineol, $\alpha$-cadinol, $\beta$-pinene, elixene, $\alpha$-pinene, and germacrene $\mathrm{D}[8,59,60]$.

4-Terpinene, $\gamma$-terpinene, $\alpha$-terpinene, and sabinene were the main compounds of EO from O. majorana [27]. Another study showed that the major chemical components of O. vulgare EO were carvacrol and terpinen-4-ol [61]. The insecticidal activity of Origanum against larvae of C. pipiens was found [62], where thymol $\left(\mathrm{LC}_{50}=36 \mathrm{mg} / \mathrm{L}\right)$ and carvacrol $\left(\mathrm{LC}_{50}=37.6 \mathrm{mg} / \mathrm{L}\right)$ were responsible for this activity.

$\alpha$-Pinene, (E)-caryophyllene, (E)-nerolidol, and cedran-8-ol were the main compounds in P. guajava leaf EO, which agreed with in one study [63], while another study identified $\alpha$-pinene and 1,8-cineole as the major components [64]. (E)-nerolidol was found in $18.5 \%$ and $17 \%$ amounts in EOs from young and mature leaves of P. guajava varieties, while $\beta$-caryophyllene was identified as a major constituent in EO from five Brazilian guava cultivars [65].

Another study showed that $(E)$-caryophyllene, caryophyllene oxide, and $\alpha$-humulene were the main compounds in the essential oil of P. guajava leaves collected from Espírito Santo, Brazil [24]. The compounds iso-caryophyllene, veridiflorene, farnesene, dl-limonene, $\Delta$-cadinene, $\alpha$-copaene, and $\alpha$-humulene were found to be abundant in the EO of plants collected from the Alsharqia region, Sultanate of Oman [66], while $\alpha$-terpinyl acetate, trans-caryophyllene, nerolidol, $\alpha$-cadinol, $\alpha$-copaene, $\alpha$-humulene, and aryphyllene oxide were found in plants collected from Northeast India [67].

\subsection{Fumigant Toxicity on T. Castaneum}

The $\mathrm{LC}_{50}$ ranged from $<2$ to $33.3 \mu \mathrm{g} / \mathrm{L}$ air for $\mathrm{EO}$ of $S$. terebinthifolius ripe fruits, $<2$ to $6.8 \mu \mathrm{g} / \mathrm{L}$ air for EO of S. terebinthifolius unripe fruits, $<2$ to $65.1 \mu \mathrm{g} / \mathrm{L}$ air for EO of S. terebinthifolius leaves, 37.9 to $>40 \mu \mathrm{g} / \mathrm{L}$ air for EO of O. majorana leaves, and $<2$ to $60.2 \mu \mathrm{g} / \mathrm{L}$ air for EO of P. guajava leaves, which means that the $\mathrm{EO}$ of $\mathrm{S}$. terebinthifolius unripe fruits had a stronger effect on T. castaneum adults than the other tested EOs using the fumigation method. Our results agree with those of Abdelgaleil et al. [68] who reported that the $\mathrm{EO}$ of $O$. vulgare $\left(\mathrm{LC}_{50}=1.6 \mu \mathrm{g} / \mathrm{L}\right.$ air $)$ was the most potent toxicant against $S$. oryzae adults. At the same time, $\mathrm{EO}$ of $S$. terebinthifolius possessed strong fumigant toxicity ( $\mathrm{LC}_{50}<30 \mathrm{mg} / \mathrm{L}$ air).

Savory and marjoram EOs had $72.5 \%$ and $67.5 \%$ mortality, respectively, on T. castaneum adults when exposed to $150 \mu \mathrm{L} / \mathrm{L}$ air for $24 \mathrm{~h} \mathrm{[69].} \mathrm{The} \mathrm{insecticidal} \mathrm{activity} \mathrm{of} \mathrm{oil} \mathrm{of} \mathrm{Origanum} \mathrm{leaves} \mathrm{in} \mathrm{a}$ vapor-phase toxicity bioassay against $T$. castaneum adults reached $\mathrm{LC}_{50}=73.7 \mu \mathrm{L} / \mathrm{L}$ air [70], while the EOs obtained from leaves and flowers showed insecticidal activity against $T$. castaneum adults [71]. Thymol and other compounds of O. majorana EO, showed insecticidal activity against $S$. oryzae and $R$. dominica adults [72].

The P. guajava treatments caused significantly higher mortality at 21 days of exposure when compared to the control. None of the treatments of P. guajava achieved 100\% mortality throughout the experimental period. Since mortality was found to be directly proportional to exposure time and concentration, increased mortality might be attained by increasing either or both [73].

For the mode of toxic action, some monoterpenes had an inhibitory effect on acetylcholinesterase activity [74,75], bound with octopamine receptors [76] and GABA-gated chloride ion channels [77].

\subsection{Mosquitocide Activity of Tested Essential Oils}

In this study, five EOs belonging to several classes was examined to compare their relative toxicity against $C$. pipiens larvae. The EOs of $O$. majorana leaves and $S$. terebinthifolius leaves and unripe fruits showed larvicidal toxicity. The tested EOs had $\mathrm{LC}_{50}$ values for the larval and adult stages under $100 \mathrm{mg} / \mathrm{L}(9.7-90.9 \mathrm{mg} / \mathrm{L})$, except for the EOs of S. terebinthifolius ripe fruits and leaves as well as $P$. guajava leaves, which had LC 50 of total larval mortality of $18,475.3,115.6$, and $1719.1 \mathrm{mg} / \mathrm{L}$, respectively. Therefore, the EOs of O. majorana leaves, S. terebinthifolius leaves, and unripe fruits EOs have potential as effective mosquitocides. In addition, the bioactivity of most monoterpenes against $C$. pipiens was evaluated in the present experiment. The leaves of the Origanum herb are rich in EO, which confers 
its characteristic and fragrance. The larval toxicity of some plant extracts, EOs, and phytochemicals against $C$. pipiens has been reported [78-81].

With the present results, total larval mortality at 10 to $100 \mathrm{mg} / \mathrm{L}$ ranged from $40.6 \%$ to $68 \%$ with EO of S. terebinthifolius unripe fruits, while it was $42.6 \%$ to $78 \%$ with EO of O. majorana leaves. Mortality increased with a growing concentration and time of exposure. The present data confirms that the EOs of $O$. majorana leaves as well as $S$. terebinthifolius unripe fruits and leaves had a more larvicidal effect than EOs of S. terebinthifolius ripe fruits and P. guajava leaves on C. pipiens at the higher concentration $(100 \mathrm{mg} / \mathrm{L})$.

The majority compounds 4-terpinene, $\gamma$-terpinene, $\alpha$-terpinene, and sabinene of $O$. majorana EO showed larvicidal activity against $C$. pipiens with $\mathrm{LC}_{50}$ and $\mathrm{LC}_{90}$ values of $258.7 \mathrm{mg} / \mathrm{L}$ and $580.4 \mathrm{mg} / \mathrm{L}$, respectively [27].

EO from O. vulgare, with the main compounds of carvacrol and terpinen-4-ol, had a significant toxic effect against early third-stage larvae of Anopheles stephensi and An. subpictus, C. quinquefasciatus, and $C$. tritaeniorhynchus, which had $\mathrm{LC}_{50}$ values of $67,74.1,80.3$, and $84.9 \mu \mathrm{g} / \mathrm{mL}$, respectively [60].

The tested EOs of S. terebinthifolius ripe/unripe fruits and leaves, O. majorana leaves, and P. guajava leaves affected larval and pupal longevity of $C$. pipiens due to prolonged larval longevity. Larval longevity at $100 \mathrm{mg} / \mathrm{L}$ was 20.6, 16.3, 18.2, 14.8, and 19.9 days, respectively. Similar to Abd El Meguid et al. [82], the toxicological activity of four plant oils including $O$. majorana had prominent mosquitocidal activity against $A$. caspius and $C$. pipiens, along with toxic effects against larvae and pupae.

The most abundant identified compound of EOs of $S$. terebinthifolia fruits and seeds was $\Delta-3$-carene and the least abundant identified compound was $\gamma$-elemene. The EOs were observed to have mosquitocidal activity against An. gambiae, An. Arabiensis, and C. quinquefasciatus. The mortality of $C$. quinquefasciatus ranged from $0.5 \%$ to $96.7 \%$, and of An. gambiae from $13.7 \%$ to $97.9 \%$ [23].

From the present results, the adult mortality ranged from $36 \%$ to $58 \%$ with EO of $S$. terebinthifolius ripe fruits, $58.6 \%$ to $94 \%$ with EO of S. terebinthifolius unripe fruits, $46 \%$ to $78 \%$ with EO of S. terebinthifolius leaves, $62.6 \%$ to $100 \%$ with EO of O. majorana leaves, and $34.6 \%$ to $63.3 \%$ with EO of P. guajava leaves at $100 \mathrm{mg} / \mathrm{L}$. The $\mathrm{LC}_{50}$ of adult C. pipiens was $65.8 \mathrm{mg} / \mathrm{L}$ for $\mathrm{EO}$ of $P$. guajava leaves. Our results align with Sowmyashree et al. [83], who reported $\mathrm{LC}_{50}$ and $\mathrm{LC}_{90}$ values of EO of P. guajava at $24 \mathrm{~h}$ of $40.2 \mathrm{ppm}$, $56.4 \mathrm{ppm}, 38 \mathrm{ppm}$, and $51.5 \mathrm{ppm}$.

From the previously identified chemical components in the tested EOs, it can be considered that they have insecticidal properties against immature stages of $C$. pipiens and the adult stage of T. castaneum.

\section{Conclusions}

The present data confirm that the essential oils of $O$. majorana leaves and S. terebinthifolius unripe fruits and leaves have more larvicidal effect than those of S. terebinthifolius ripe fruits and P. guajava leaves on C. pipiens at a higher concentration $(100 \mathrm{mg} / \mathrm{L})$ when applied by the dipping method. Additionally, EOs of $S$. terebinthifolius unripe and ripe fruits, P. guajava leaves, and S. terebinthifolius leaves have more adulticidal effect than $O$. majorana leaf oil against $T$. castaneum when applied by the fumigant method.

Author Contributions: A.M.E.-S. and M.Z.M.S. designed the experiment and carried out the methodology and laboratory analyses. M.B.-J. and A.A.A. contributed the reagents, materials, and analytical tools. All authors shared in writing and revising the paper.

Acknowledgments: This research was funded by the Deanship of Scientific Research at Princess Nourah bint Abdulrahman University through the Fast-track Research Funding Program.

Conflicts of Interest: The authors declare no conflict of interest. 


\section{References}

1. Cavalcanti, E.S.B.; Morais, S.M.; Lima, M.A.A.; Santana, E.W.P. Larvicidal activity of essential oils from Brazilian plants against Aedes aegypti L. Mem. Inst. Oswaldo Cruz 2004, 99, 541-544. [CrossRef] [PubMed]

2. Cheng, S.; Huang, C.; Chen, Y.; Yu, J.; Chen, W.; Chang, S. Chemical compositions and larvicidal activities of leaf essential oils from two eucalyptus species. Biores. Technol. 2009, 100, 452-456. [CrossRef] [PubMed]

3. Farag, M.; Ahmed, M.H.M.; Yousef, H.; Abdel-Rahman, A.A.H. Repellent and insecticidal activities of Melia azedarach L. against cotton leafworm, Spodoptera littoralis (Boisd.). Z. Naturforsch. 2011, 66, 129-135. [CrossRef] [PubMed]

4. Rajamma, A.J.; Dubey, S.; Sateesha, S.B.; Tiwari, S.N.; Ghosh, S.K. Comparative larvicidal activity of different species of Ocimum against Culex quinquefasciatus. Nat. Prod. Res. 2011, 25, 1916-1922. [CrossRef] [PubMed]

5. Ghosh, A.; Chowdhury, N.; Chandra, G. Plant extracts as potential mosquito larvicides. Indian J. Med. Res. 2012, 135, 581-598. [PubMed]

6. Govindarajan, M.; Sivakumar, R.; Rajeswary, M.; Yogalakshmi, K. Chemical composition and larvicidal activity of essential oil from Ocimum basilicum (L.) against Culex tritaeniorhynchus Aedes albopictus and Anopheles subpictus (Diptera: Culicidae). Exp. Parasitol. 2013, 134, 7-11. [CrossRef] [PubMed]

7. Elansary, H.O.; Salem, M.Z.M.; Ashmawy, N.A.; Yessoufou, K.; El-Settawy, A.A. In vitro antibacterial, antifungal, and antioxidant activities of Eucalyptus spp. leaf extracts related to phenolic composition. Nat. Prod. Res. 2017, 31, 2927-2930. [CrossRef]

8. Hussein, H.S.; Salem, M.Z.M.; Soliman, A.M. Repellent, attractive, and insecticidal effects of essential oils from Schinus terebinthifolius fruits and Corymbia citriodora leaves on two whitefly species, Bemisia tabaci and Trialeurodes ricini. Sci. Horticul. 2017, 216, 111-119. [CrossRef]

9. Hamada, H.M.; Awad, M.; El-Hefny, M.; Moustafa, M.A.M. Insecticidal activity of garlic (Allium sativum) and ginger (Zingiber officinale) oils on the cotton leafworm, Spodoptera littoralis (Boisd.) (Lepidoptera: Noctuidae). Afr. Entomol. 2018, 26, 84-94. [CrossRef]

10. Hamad, Y.K.; Abobakr, Y.; Salem, M.Z.M.; Ali, H.M.; Al-Sarar, A.S.; Al-Zabib, A.A. Activity of plant extracts/essential oils against three plant pathogenic fungi and mosquito larvae: GC/MS Analysis of Bioactive Compounds. BioResources 2019, 14, 4489-4511.

11. Isman, M.B.; Machial, C.; Miresmailli, S.; Bainard, L. Essential oil-based pesticides: New insights from old chemistry. In Pesticide Chemistry; Ohkawa, H., Miyagawa, H., Lee, P., Eds.; Wiley-VCH: Weinheim, Germany, 2007; pp. 201-209.

12. Abdelgaleil, S.A.M.; Mohamed, M.I.E.; Badawy, M.E.I.; El-arami, S.A.A. Fumigant and contact toxicities of monoterpenes to Sitophilus oryzae (L.) and Tribolium castaneum (Herbst) and their inhibitory effects on acetylcholinesterase activity. J. Chem. Ecol. 2009, 35, 518-525. [CrossRef] [PubMed]

13. Abdelsalam, N.R.; Salem, M.Z.M.; Ali, H.M.; Mackled, M.I.; EL-Hefny, M.; Elshikh, M.S.; Hatamleh, A.A. Morphological, biochemical, molecular, and oil toxicity properties of Taxodium trees from different locations. Ind. Crops Prod. 2019, 139, 111515. [CrossRef]

14. Wang, C.F.; Yang, K.; You, C.X.; Zhang, W.J.; Guo, S.S.; Geng, Z.F.; Du, S.S.; Wang, Y.Y. Chemical composition and insecticidal activity of essential oils from Zanthoxylum dissitum leaves and roots against Three Species of Storage Pests. Molecules 2015, 20, 7990-7999. [CrossRef]

15. Wahba, T.F.; Mackled, M.I.; Selim, S.; El-Zemity, S.R. Toxicity and reproduction inhibitory effects of some monoterpenes against the cowpea weevil Callosobruchus maculatus F. (Coleoptera: Chrysomelidae: Bruchinae). Middle East J. Appl. Sci. 2018, 8, 1061-1070.

16. Russell, T.L.; Kay, B.H.; Skilleter, G.A. Environmental effects of mosquito insecticides on saltmarsh invertebrate fauna. Aquat. Biol. 2009, 6, 77-90. [CrossRef]

17. Alkenani, N.A. Influence of the mixtures composed of slow-release insecticide formulations against Aedes aegypti mosquito larvae reared in pond water. Saudi J. Biol. Sci. 2017, 24, 1181-1185. [CrossRef]

18. Murray, B.I. Botanical insecticides, deterrents and repellents in modern and an increasingly regulated world. Annu. Rev. Entomol. 2006, 51, 45-66.

19. Chandler, D.; Bailey, A.S.; Tatchell, G.; Davidson, G.; Greaves, J.; Grant, W.P. The development, regulation and use of biopesticides for integrated pest management. Philos. Trans. R. Soc. B Biol. Sci. 2011, 366, 1987-1998. [CrossRef] 
20. Silva, A.G.; Almeida, D.L.; Ronchi, S.N.; Bento, A.C.; Scherer, R.; Ramos, A.C.; Cruz, Z.M.A. The essential oil of Brazilian pepper, Schinus terebinthifolia Raddi in larval control of Stegomyia aegypti (Linnaeus, 1762). Parasites Vectors 2010, 3, 79. [CrossRef]

21. Ennigrou, A.; Casabianca, H.; Laarif, A.; Hanchi, B.; Hosni, K. Maturation-related changes in phytochemicals and biological activities of the Brazilian pepper tree (Schinus terebinthifolius Raddi) fruits. S. Afr. J. Bot. 2017, 108, 407-415. [CrossRef]

22. Procópio, T.F.; Fernandes, K.M.; Pontual, E.V.; Ximenes, R.M.; Oliveira, A.R.; Souza, C.S.; Albuquerque Melo, A.M.; Amaral Ferraz Navarro, D.M.; Paiva, P.M.; Martins, G.F.; et al. Schinus terebinthifolius leaf extract causes midgut damage, interfering with survival and development of Aedes aegypti larvae. PLoS ONE 2015, 10, e0126612. [CrossRef]

23. Kweka, E.J.; Nyindo, M.; Mosha, F.; Silva, A.G. Insecticidal activity of the essential oil from fruits and seeds of Schinus terebinthifolia Raddi against African malaria vectors. Parasites Vectors 2011, 4, 129. [CrossRef]

24. Mendes, L.A.; Martins, G.F.; Valbon, W.R.; da Silva de Souza, T.; Menini, L.; Ferreira, A.; da Silva Ferreira, M.F. Larvicidal effect of essential oils from Brazilian cultivars of guava on Aedes aegypti L. Ind. Crops Prod. 2017, 108, 684-689. [CrossRef]

25. Lima, M.A.A.; Oliveira, F.M.M.; Gomes, G.A.; Lavor, P.L.; Santiago, G.M.P.; Nagao-Dias, A.T.; Arriaga, A.M.C.; Lemos, T.L.G.; Carvalho, M.G. Evaluation of larvicidal activity of the essential oils of plants species from Brazil against Aedes aegypti (Diptera: Culicidae). Afr. J. Biotechnol. 2011, 10, 11716-11720.

26. Satyal, P.; Paudel, P.; Lamichhane, B.; Setzer, W.N. Leaf essential oil composition and bioactivity of Psidium guajava from Kathmandu, Nepal. Amer. J. Essen. Oils Nat. Prod. 2015, 3, 11-14.

27. El-Akhal, F.; Lalami, A.E.O.; Zoubi, Y.E.; Greche, H.; Guemmouh, R. Chemical composition and larvicidal activity of essential oil of Origanum majorana (Lamiaceae) cultivated in Morocco against Culex pipiens (Diptera: Culicidae). Asian Pac. J. Trop. Biomed. 2014, 4, 746-750. [CrossRef]

28. Azizi, A.; Yan, F.; Honermeier, B. Herbage yield, essential oil content and composition of three oregano (Origanum vulgare L.) populations as affected by soil moisture regimes and nitrogen supply. Ind. Crops Prod. 2009, 29, 554-561. [CrossRef]

29. Pavela, R. Larvicidal effects of various Euro-Asiatic plants against Culex quinquefasciatus Say larvae (Diptera: Culicidae). Parasitol. Res. 2008, 102, 555-559. [CrossRef]

30. Zahran, H.A.; Abdelgaleil, S.A.M. Insecticidal and developmental inhibitory properties of monoterpenes on Culex pipiens L. (Diptera: Culicidae). J. Asia Pac. Entomol. 2011, 14, 46-51.

31. WHO. World Malaria Report 2016; WHO Press: Geneva, Switzerland, 2016.

32. Rajashekar, Y.; Bakthavatsalam, N.; Shivanandappa, T. Botanicals as Grain Protectants. Psyche 2012, 2012, 646740. [CrossRef]

33. Talukder, F.A. Plant products as potential stored product insect management agents-A mini review. Emir. J. Agric. Sci. 2000, 18, 17-32. [CrossRef]

34. Obeng-Ofori, D.; Reichmuth, C. Bioactivity of eugenol, a major component of essential oil of Ocimum suave (Wild.) against four species of stored-product Coleopteran. Inter. J. Pest Manag. 1997, 43, 89-94. [CrossRef]

35. Asawalam, E.F.; Adesiyan, S.O. Potentials of Ocimum basilicum (Linn.) for the control of Sitophilus zeamais (Motsch). Niger. Agric. J. 2001, 32, 195-201.

36. Rajashekar, Y.; Gunasekaran, N.; Shivanandappa, T. Insecticidal activity of the root extract of Decalepis hamiltonii against stored-product insect pests and its application in grain protection. J. Food Sci. Technol. 2010, 47, 310-314. [CrossRef] [PubMed]

37. Devi, K.C.; Devi, S.S. Insecticidal and oviposition deterrent properties of some spices against coleopteran beetle, Sitophilus oryzae. J. Food Sci. Technol. 2011, 50, 600-604. [CrossRef] [PubMed]

38. Rajendran, S.; Sriranjini, V. Plant products as fumigants for stored-product insect control. J. Stored Prod. Res. 2008, 44, 126-135. [CrossRef]

39. Mackled, M.I.; EL-Hefny, M.; Bin-Jumah, M.; Wahba, T.F.; Allam, A.A. Assessment of the toxicity of natural oils from Mentha piperita, Pinus roxburghii, and Rosa spp. against three stored product insects. Processes 2019, 7, 861. [CrossRef]

40. Okla, M.K.; Alamri, S.A.; Salem, M.Z.M.; Ali, H.M.; Behiry, S.I.; Nasser, R.A.; Alaraidh, I.A.; Al-Ghtani, S.M.; Soufan, W. Yield, phytochemical constituents, and antibacterial activity of essential oils from the leaves/twigs, branches, branch wood, and branch bark of Sour Orange (Citrus aurantium L.). Processes 2019, 7, 363. [CrossRef] 
41. Salem, M.Z.M.; Elansary, H.O.; Ali, H.M.; El-Settawy, A.A.; Elshikh, M.S.; Abdel-Salam, E.M.; Skalicka-Woźniak, K. Antibacterial, antifungal, and antioxidant activities of essential oils extracted from Cupressus macrocarpa branchlets and Corymbia citriodora leaves grown in Egypt. BMC Complement. Alter. Med. 2018, 18, 23. [CrossRef]

42. Salem, M.Z.M.; EL-Hefny, M.; Ali, H.M.; Elansary, H.O.; Nasser, R.A.; El-Settawy, A.A.A.; El Shanhorey, N.; Ashmawy, N.A.; Salem, A.Z.M. Antibacterial activity of extracted bioactive molecules of Schinus terebinthifolius ripened fruits against some pathogenic bacteria. Microb. Pathogen. 2018, 120, 119-127. [CrossRef]

43. Adams, R.P. Identification of Essential Oil Components by Gas Chromatograph/Quadruple Mass Spectroscopy; Allured Publishing: Carol Stream, IL, USA, 1995; p. 456.

44. Davies, N.W. Gas chromatographic retention indices of monoterpenes and sesquiterpenes on methyl silicone and Carbowax 20M phases. J. Chromatog. A 1990, 503, 1-24. [CrossRef]

45. Salem, M.Z.M.; Mansour, M.M.A.; Elansary, H.O. Evaluation of the effect of inner and outer bark extracts of Sugar Maple (Acer saccharum var. saccharum) in combination with citric acid against the growth of three common molds. J. Wood Chem. Technol. 2019, 39, 136-147. [CrossRef]

46. Salem, M.Z.M.; Behiry, S.I.; EL-Hefny, M. Inhibition of Fusarium culmorum, Penicillium chrysogenum and Rhizoctonia solani by $n$-hexane extracts of three plant species as a wood-treated oil fungicide. J. Appl. Microbiol. 2019, 126, 1683-1699. [CrossRef]

47. Mohamed, W.A.; Mansour, M.M.A.; Salem, M.Z.M. Lemna gibba and Eichhornia crassipes extracts: Clean alternatives for deacidification, antioxidation and fungicidal treatment of historical paper. J. Clean. Prod. 2019, 219, 846-855. [CrossRef]

48. EL-Hefny, M.; Ashmawy, N.A.; Salem, M.Z.M.; Salem, A.Z.M. Antibacterial activity of the phytochemicals-characterized extracts of Callistemon viminalis, Eucalyptus camaldulensis and Conyza dioscoridis against the growth of some phytopathogenic bacteria. Microb. Pathogen. 2017, 113, 348-356. [CrossRef] [PubMed]

49. Finney, D.J. Probit Analysis, 2nd ed.; Cambridge University Press: Cambridge, London, UK, 1971; p. 318.

50. El-Bakry, A.M.; Abdel-Aziz, N.F.; Sammour, E.A.; Abdelgaleil, S.A.M. Insecticidal activity of natural plant essential oils against some stored product insects and their side effects on wheat seed germination. Egypt. J. Biol. Pest Cont. 2016, 26, 83-88.

51. Huang, Y.; Lam, S.L.; Ho, S.H. Bioactivities of essential oil from Elletaria cardamomum (L.) Maton. to Sitophilus zeamais Motschulsky and Tribolium castaneum (Herbst). J. Stored Prod. Res. 2000, 36, 107-117. [CrossRef]

52. SAS. Users Guide: Statistics (Release 8.02); SAS Inst Inc.: Cary, NC, USA, 2001.

53. Barbosa, L.C.A.; Demuner, A.J.; Clemente, A.D.; Fonseca de Paula, V.; Ismail, F.M.D. Seasonal variation in the composition of volatile oils from Schinus terebinthifolius Raddi. Quim. Nova 2007, 30, 1959-1965. [CrossRef]

54. Atti dos Santos, A.C.; Rossato, M.; Agostini, F.; Atti Serafini, L.; Luciana dos Santos, P.; Molon, R.; Dellacassa, E.; Moyna, P. Chemical composition of the essential oils from leaves and fruits of Schinus molle L. and Schinus terebinthifolius Raddi from Southern Brazil. J. Essen. Oil Bear. Plant. 2009, 12, 16-25. [CrossRef]

55. Cole, E.R.; dos Santos, R.B.; Lacerda Júnior, V.; Martins, J.D.L.; Greco, S.J.; Cunha Neto, A. Chemical composition of essential oil from ripe fruit of Schinus terebinthifolius Raddi and evaluation of its activity against wild strains of hospital origin. Braz. J. Microbiol. 2014, 45, 821-828. [CrossRef]

56. Périno-Issartier, S.; Abert-Vian, M.; Petitcolas, E.; Chemat, F. Microwave turbo hydrodistillation for rapid extraction of the essential oil from Schinus terebinthifolius Raddi berries. Chromatographia 2010, 72, 347-350. [CrossRef]

57. Richter, R.; von Reuß, S.H.; König, W.A. Spirocyclopropane-type sesquiterpene hydrocarbons from Schinus terebinthifolius Raddi. Phytochemistry 2010, 71, 1371-1374. [CrossRef] [PubMed]

58. Bendaoud, H.; Romdhane, M.; Souchard, J.P.; Cazaux, S.; Bouajila, J. Chemical composition and anticancer and antioxidant activities of Schinus Molle, L. and Schinus terebinthifolius Raddi berries essential oils. J. Food Sci. 2010, 75, 466-472. [CrossRef] [PubMed]

59. El-Shazli, E.M.; Hafezh, S.S.; Abdel-Ghany, A.E. Analysis of the essential oils of S. terebinthifolius Raddi cultivated in Egypt. Zag. J. Pharm. Sci. 2000, 9, 1-8. 
60. Ibrahim, M.T.; Fobbe, R.; Nolte, J. Chemical composition and biological studies of Egyptian Schinus molle L. and Schinus terebinthifolius Raddi oils. Bull. Facul Pharm. Cairo Univ. 2004, 42, 289.

61. Govindarajan, M.; Rajeswary, M.; Hoti, S.L.; Benelli, G. Larvicidal potential of carvacrol and terpinen-4-ol from the essential oil of Origanum vulgare (Lamiaceae) against Anopheles stephensi, Anopheles subpictus, Culex quinquefasciatus and Culex tritaeniorhynchus (Diptera: Culicidae). Res. Veter. Sci. 2016, 104, 77-82. [CrossRef]

62. Traboulsi, A.F.; Taoubi, K.; El-Haj, S.; Bessiere, J.M.; Rammal, S. Insecticidal properties of essential plant oils against the mosquito Culex pipiens molestus (Diptera: Culicideae). Pest Manag. Sci. 2002, 58, 491-495. [CrossRef]

63. Pino, J.A.; Agüero, J.; Marbot, R.; Fuentes, V. Leaf oil of Psidium guajava L. from Cuba. J. Essen. Oil Res. 2001, 13, 61-62. [CrossRef]

64. Ji, X.D.; Pu, Q.L.; Garraffo, H.M.; Pannell, L.K. The Essential oil of the leaves of Psidium guajava L. J. Essen. Oil Res. 1991, 3, 187-189. [CrossRef]

65. Silva, J.A.A.; Hall, D.G.; Gottwald, T.R.; Andrade, M.S.; Maldonado, W., Jr.; Alessandro, R.T.; Lapointe, S.L.; Andrade, E.C.; Machado, M.A. Repellency of selected Psidium guajava cultivars to the Asian citrus psyllid, Diaphorina citri. Crop Protec. 2016, 84, 14-20. [CrossRef]

66. Weli, A.; Al-Kaabi, A.; Al-Sabahi, J.; Said, S.; Hossain, M.; Al-Riyami, S. Chemical composition and biological activities of the essential oils of Psidium guajava leaf. J. King Saud Univ. Sci. 2018. [CrossRef]

67. Borah, A.; Pandey, S.K.; Haldar, S.; Lal, M. Chemical composition of leaf essential oil of Psidium guajava L. from North East India. J. Essen. Oil Bear. Plant. 2019, 22, 248-253. [CrossRef]

68. Abdelgaleil, S.A.M.; Mohamed, M.I.E.; Shawir, M.S.; Abou-Taleb, H.K. Chemical composition, insecticidal and biochemical effects of essential oils of different plant species from Northern Egypt on the rice weevil, Sitophilus oryzae L. J. Pestic. Sci. 2016, 89, 219-229. [CrossRef]

69. Karabörklü, S.; Ayvaz, A.; Yilmaz, S. Bioactivities of different essential oils against the adults of two stored product insects. Pak. J. Zool. 2010, 42, 679-686.

70. Salaheddine, S.; Zohra, B.; Cheikh, I.C.; Asma, L. Study of the toxicity of essential oils of Origanum majorana on Tribolium castaneum and Plodia interpunctella (stored product insects). Tunis. J. Med. Plants Nat. Prod. 2013, 9, 29-34.

71. Rozman, V.; Kalinovic, I.; Korunic, Z. Toxicity of naturally occurring compounds of Lamiaceae and Lauraceae to three stored-product insects. J. Stored Prod. Res. 2007, 43, 349-355. [CrossRef]

72. Karan, T.; Simsek, S.; Yildiz, I.; Erenler, R. Chemical composition and insecticidal activity of Origanum syriacum L. essential oil against Sitophilus oryzae and Rhyzopertha dominica. Int. J. Sec. Metab. 2018, 5, 87-93.

73. Iram, N.; Arshad, M.; Akhter, N. Evaluation of Botanical and Synthetic Insecticide for the Control of Tribolium castaneum (Herbst) (Coleoptera: Tenebrionidae). Soc. Entomol. Bras. BioAssay 2013, 8, 3.

74. Miyazawa, M.; Watanabe, H.; Kameoka, H. Inhibition of acetylcholinesterase activity by monoterpenoids with a P-menthane skeleton. J. Agric. Food Chem. 1997, 45, 677-679. [CrossRef]

75. Picollo, M.I.; Toloza, A.C.; Mougabure Cueto, G.; Zygadlo, J.; Zerba, E. Anticholinesterase and pediculicidal activities of monoterpenoids. Fitoterapia 2008, 79, 271-278. [CrossRef]

76. Enan, E. Insecticidal activity of essential oils: Octopaminergic sites of action. Comp. Biochem. Physiol. C Toxicol. Pharmacol. 2001, 130, 325-337. [CrossRef]

77. Hold, K.M.; Sirisoma, N.S.; Ikeda, T.; Narahashi, T.; Casida, J.E. R-Thujone (the active component of absinthe): $\gamma$-Aminobutyric acid type A receptor modulation and metabolic detoxification. Proc. Natl. Acad. Sci. USA 2000, 97, 3826-3831. [CrossRef] [PubMed]

78. Abdelgaleil, S.A.M. Chemical composition, insecticidal and fungicidal activities of essential oils isolated from Mentha microphylla and Lantana camara growing in Egypt. Alex. Sci. Exch. J. 2006, 27, 18-28.

79. Michaelakis, A.; Mihou, A.P.; Koliopoulos, G.; Couladouros, E.A. Attract-and-kill strategy. Laboratory studies on hatched larvae of Culex pipiens. Pest Manag. Sci. 2007, 63, 954-959. [CrossRef]

80. Kim, N.J.; Byun, S.G.; Cho, J.E.; Chung, K.; Ahn, Y.J. Larvicidal activity of Kaempferia galangal rhizome phenylpropanoids towards three mosquito species. Pest Manag. Sci. 2008, 64, 857-862.

81. Radwan, M.A.; El-Zemity, S.R.; Mohamed, S.A.; Sherby, S.M. Larvicidal activity of some essential oils, monoterpenoids and their corresponding N-methyl carbamate derivatives against Culex pipiens (Diptera: Culicidae). Int. J. Trop. Insect Sci. 2008, 28, 61-68. [CrossRef] 
82. Abd El Meguid, A.D.; Mahmoud, S.H.; Baz, M.M. Toxicological activity of four plant oils against Aedes caspius and Culex pipiens (Diptera: Culicidae). Int. J. Mosq. Res. 2019, 6, 86-94.

83. Sowmyashree, K.; Chalannavar, R.K.; Ghosh, S.K.; Nityasree, B.R.; Supriya, S. Effect of essential oils of Aegle marmelos (L.) correa and Psidium guajava L. on larvae of malaria vector Anopheles stephensi Liston. Res. J. Life Sci Bioinform. Pharm. Chem. Sci. 2019, 5, 705. [CrossRef]

(C) 2019 by the authors. Licensee MDPI, Basel, Switzerland. This article is an open access article distributed under the terms and conditions of the Creative Commons Attribution (CC BY) license (http://creativecommons.org/licenses/by/4.0/). 